\title{
Marked Ethnic, Nativity, and Socioeconomic Disparities in Disability and Health Insurance among US Children and Adults: The 2008-2010 American Community Survey
}

\author{
Gopal K. Singh ${ }^{1}$ and Sue C. Lin $^{2}$ \\ ${ }^{1}$ US Department of Health and Human Services, Health Resources and Services Administration, Maternal and Child Health Bureau, \\ 5600 Fishers Lane, Room 18-41, Rockville, MD 20857, USA \\ ${ }^{2}$ US Department of Health and Human Services, Health Resources and Services Administration, Bureau of Primary Health Care, \\ 5600 Fishers Lane, Room 6A-55, Rockville, MD 20857, USA
}

Correspondence should be addressed to Gopal K. Singh; gsingh@hrsa.gov

Received 30 April 2013; Accepted 4 September 2013

Academic Editor: Anna Karakatsani

Copyright (C) 2013 G. K. Singh and S. C. Lin. This is an open access article distributed under the Creative Commons Attribution License, which permits unrestricted use, distribution, and reproduction in any medium, provided the original work is properly cited.

\begin{abstract}
We used the 2008-2010 American Community Survey Micro-data Sample $(N=9,093,077)$ to estimate disability and health insurance rates for children and adults in detailed racial/ethnic, immigrant, and socioeconomic groups in the USA. Prevalence and adjusted odds derived from logistic regression were used to examine social inequalities. Disability rates varied from $1.4 \%$ for Japanese children to $6.8 \%$ for Puerto Rican children. Prevalence of disability in adults ranged from 5.6\% for Asian Indians to $22.0 \%$ among American Indians/Alaska Natives. More than $17 \%$ of Korean, Mexican, and American Indian children lacked health insurance, compared with $4.1 \%$ of Japanese and 5.9\% of white children. Among adults, Mexicans (43.6\%), Central/South Americans (41.4\%), American Indians/Alaska Natives (32.7\%), and Pakistanis (29.3\%) had the highest health-uninsurance rates. Ethnic nativity disparities were considerable, with $58.3 \%$ of all Mexican immigrants and 34.0\% of Mexican immigrants with disabilities being uninsured. Socioeconomic gradients were marked, with poor children and adults having 3-6 times higher odds of disability and uninsurance than their affluent counterparts. Socioeconomic differences accounted for $24.4 \%$ and $60.2 \%$ of racial/ethnic variations in child health insurance and disability and $75.1 \%$ and $89.7 \%$ of ethnic inequality in adult health insurance and disability, respectively. Health policy programs urgently need to tackle these profound social disparities in disability and healthcare access.
\end{abstract}

\section{Introduction}

The racial/ethnic composition of the US population has undergone substantial change in recent decades $[1,2]$. The proportion of the White population in the US declined from $87.6 \%$ in 1970 to $63.3 \%$ in 2011, whereas the percentage of Black population increased slightly from $11.1 \%$ to $12.2 \%$ during the same time period $[1,2]$. On the other hand, the Hispanic population increased rapidly from 9.1 million (4.5\%) in 1970 to 51.9 million (16.7\%) in 2011, whereas the Asian/Pacific Islander population increased nearly 5-fold, from 3.7 million (1.6\%) in 1980 to 18.2 million (5.8\%) in 2011 [1-5]. Changes in the racial/ethnic composition have occurred primarily as a result of large-scale immigration from Latin America and Asia during the past four decades
[6-9]. The immigrant population grew from 9.6 million in 1970 to 40.4 million in 2011 [2, 6-9]. Immigrants currently represent $13.0 \%$ of the total US population [2]. Over $80 \%$ of all US immigrants currently hail from Latin America and Asia, in contrast to 1960 when Europeans accounted for $75 \%$ of the foreign-born population [6-8]. Increase in the number of immigrant children has also been substantial, with the number doubling from 8.2 million in 1990 to 17.5 million in $2011[2,10]$. In 2011, nearly a quarter of US children had at least one foreign-born parent $[2,10]$.

Despite such marked increases in the immigrant population and growing ethnic heterogeneity of the US population, analysis of health inequalities according to detailed ethnic and national origins, particularly among recent ethnic 
and immigrant groups from Asia such as those from the Indian subcontinent, Korea, Vietnam, Laos, Cambodia, and Thailand, remains relatively uncommon [11-17]. Besides the 2000 and prior decennial censuses, the American Community Survey (ACS) is the only contemporary national data source in the USA that provides extensive socioeconomic, demographic, disability, and health insurance information for a large number of ethnic groups and countries of origin, including some of the newly arrived ethnic groups from Asia, Africa, Latin America, and the Caribbean [1, 2, 6, 13-20].

Disability is a major morbidity and health status indicator both in the United States and globally [21-24]. More than a billion people, about $15 \%$ of the world's population, are estimated to have some form of disability [21]. Disability rates have been rising in many countries of the world due to population aging and increases in chronic health conditions [21]. In 2011, an estimated 37.2 million people (12\%) in the US had disability [2]. In the USA and across the world, people with disabilities are more likely to report poorer physical and mental health status, higher rates of smoking, physical inactivity, obesity, and alcohol use, lower income and educational achievements, higher poverty and unemployment rates, and experiencing more barriers in accessing social, economic, transport, and healthcare services than people without disabilities [2, 21, 23, 25]. Health insurance coverage is a major determinant of access to healthcare [22]. Although in much of the industrialized world, healthcare coverage is generally available to all citizens, 46.4 million Americans, including 5.5 million US children, were without health insurance in $2011[2,26]$. Research has shown that uninsured individuals are much more likely to delay or forego preventive health services and needed medical care, have higher rates of mortality, and are more likely to be diagnosed with an advanced stage disease than individuals with health insurance [22, 26-28].

Although previous research has examined racial/ethnic and nativity disparities in disability rates in the USA using the 1990 and 2000 decennial censuses, disability and health uninsurance rates have not been analyzed for both children and adults from detailed ethnic and immigrant groups [1317]. Although substantial ethnic, nativity, and socioeconomic inequalities in health, life expectancy, all-cause and causespecific mortality, and chronic disease conditions are well documented, such inequalities in disability have been less well studied [11-17, 22, 29-32]. Analyzing social inequalities in disability is important because ethnic and socioeconomic characteristics can significantly influence factors underlying the disablement process, including the development of physical and mental impairments, comorbidities, health-risk behaviors, and performance of social roles and activities in relation to family, work, or independent living $[33,34]$. Social inequalities research can also help identify vulnerable groups, including ethnic minority, immigrant, low-income, and socially disadvantaged groups, who are at high risk of disability and uninsurance and who could benefit from public policy and social interventions designed to reduce the impact of disability and uninsurance. Moreover, emphasis on ethnicity and socioeconomic factors is consistent with the national health initiative, Healthy People 2020, which calls for further reductions or elimination of social inequalities in health, disease, disability, and access to health services [35].

In this study, we use a recent three-year pooled ACS sample containing more than 9 million people to estimate child and adult disability and health insurance rates for detailed racial/ethnic, nativity, and socioeconomic groups in the USA and examine ethnic and nativity patterns after controlling for socioeconomic and demographic characteristics. Additionally, we examine ethnic and socioeconomic disparities in health insurance coverage among people with disabilities.

\section{Methods}

Data for the present analysis came from the 2008-2010 ACS Micro-data Sample [36]. Decennial censuses conducted by the US Census Bureau have long been the source of detailed socioeconomic and demographic information for various ethnic and immigrant populations in the United States [1]. With the discontinuation of the long-form questionnaire in the 2010 decennial census, the ACS has become the primary census database for producing socioeconomic, demographic, housing, and labor force characteristics of various population groups, including ethnic and immigrant populations, at the national, state, county, and local levels $[2,37]$. The advantage of the ACS is that it is conducted annually with a sample size of over 3 million records, as compared with the decennial census long-form data, which were only available every 10 years [37]. The ACS uses a complex, multistage probability design and is representative of the civilian noninstitutionalized population, covering all communities in the USA [36-38]. The household response rate for the 2008-2010 ACS was $98 \%$ $[2,38]$. All data are based on self-reports and obtained via mail-back questionnaire, telephone, and in-home personal interviews $[36,37]$. Substantive and methodological details of the ACS are described elsewhere [36-38].

2.1. Dependent Variables. Analyses of the two dependent variables, disability and health insurance, were carried out for 9,093,077 individuals, including 2.1 million children aged $<18$ years. Disability status was a dichotomous variable which defined an individual having a disability if s/he reported serious vision, hearing, cognitive, ambulatory, self-care, or independent living difficulties [36]. The ACS concept of disability captures these six aspects of disability to define an overall measure or specific disability types $[18,19,36]$. To derive vision-related disability, the ACS respondents are asked if they are "blind or ... have serious difficulty seeing even when wearing glasses." Hearing difficulty is derived from a question that asks respondents if they are "deaf or ... have serious difficulty hearing." Cognitive difficulty involves serious difficulty concentrating, remembering, or making decisions due to a physical, metal, or emotion condition. Ambulatory difficulty is based on a question that asks respondents if they have "serious difficulty walking or climbing stairs." Selfcare difficulty is based on the question whether or not the respondent has difficulty dressing or bathing. Independent living difficulty is determined if the respondent reports having difficulty doing errands alone such as visiting a doctor's office 
TABLE 1: Descriptive socioeconomic and demographic characteristics for major racial/ethnic groups in the United States: the 2008-2010 American Community Survey $(N=9,093,077)$.

\begin{tabular}{|c|c|c|c|c|c|c|c|c|c|}
\hline Racial/ethnic group & $\begin{array}{c}\text { Number } \\
\text { in sample }\end{array}$ & $\begin{array}{c}\text { Population } \\
\text { proportion } \\
(\%)\end{array}$ & $\begin{array}{c}\text { Child } \\
\text { poverty } \\
\text { rate }(\%)\end{array}$ & $\begin{array}{c}\text { Adult } \\
\text { poverty }^{2} \\
\text { rate }(\%)\end{array}$ & $\begin{array}{c}\text { Per capita } \\
\text { income }^{2} \\
(\$)\end{array}$ & $\begin{array}{c}\text { College } \\
\text { graduates } \\
(\%)\end{array}$ & $\begin{array}{c}\text { Unemploy- } \\
\text { ment rate } \\
(\%)\end{array}$ & $\begin{array}{c}\text { Immigrant } \\
\text { population } \\
(\%)\end{array}$ & $\begin{array}{c}\text { Divorced or } \\
\text { separated }^{2} \\
(\%)\end{array}$ \\
\hline United States & $9,093,077$ & 100.00 & 19.96 & 12.48 & 36,498 & 28.03 & 8.63 & 12.82 & 13.54 \\
\hline Non-Hispanic White & $6,335,580$ & 64.14 & 11.97 & 9.42 & 39,964 & 31.11 & 7.20 & 3.84 & 13.53 \\
\hline Mexican & 780,939 & 10.45 & 32.10 & 20.65 & 22,171 & 9.25 & 10.25 & 36.19 & 10.99 \\
\hline Puerto Rican & 108,418 & 1.49 & 32.11 & 21.60 & 26,896 & 16.26 & 12.99 & 1.10 & 16.51 \\
\hline Cuban & 46,579 & 0.57 & 16.05 & 15.80 & 30,992 & 24.54 & 10.67 & 58.87 & 16.45 \\
\hline Central/South American & 196,267 & 2.79 & 24.21 & 16.80 & 25,543 & 18.71 & 9.95 & 64.02 & 13.22 \\
\hline Other Hispanics & 65,309 & 0.77 & 22.95 & 15.34 & 30,267 & 21.29 & 9.80 & 13.42 & 15.51 \\
\hline Non-Hispanic Black & 903,942 & 12.23 & 36.00 & 21.40 & 25,155 & 17.82 & 14.54 & 7.85 & 17.53 \\
\hline American Indian/AN & 68,673 & 0.66 & 32.45 & 23.78 & 23,721 & 13.85 & 14.29 & 1.10 & 17.63 \\
\hline Asian Indian & 72,611 & 0.90 & 7.11 & 8.74 & 53,639 & 71.25 & 6.72 & 72.19 & 3.32 \\
\hline Chinese & 95,691 & 1.09 & 10.40 & 13.62 & 42,567 & 52.60 & 6.50 & 69.24 & 5.87 \\
\hline Filipino & 71,631 & 0.82 & 5.09 & 5.67 & 36,745 & 48.64 & 6.58 & 66.67 & 8.20 \\
\hline Japanese & 23,414 & 0.25 & 6.44 & 8.27 & 44,550 & 47.55 & 4.02 & 41.15 & 9.70 \\
\hline Korean & 38,039 & 0.47 & 13.50 & 15.35 & 35,604 & 52.02 & 7.03 & 73.91 & 6.65 \\
\hline Vietnamese & 42,879 & 0.51 & 16.54 & 14.10 & 29,415 & 26.14 & 8.20 & 68.79 & 7.84 \\
\hline Cambodian & 5,782 & 0.08 & 24.64 & 17.04 & 22,500 & 13.65 & 11.39 & 59.51 & 9.39 \\
\hline Bangladeshi & 2,523 & 0.03 & 28.68 & 21.15 & 26,887 & 49.83 & 7.95 & 73.50 & 2.50 \\
\hline Pakistani & 8,256 & 0.11 & 19.92 & 14.11 & 38,113 & 54.43 & 8.18 & 67.54 & 3.59 \\
\hline Hmong & 4,947 & 0.08 & 35.65 & 24.23 & 18,411 & 12.90 & 12.21 & 43.72 & 5.63 \\
\hline Laotian & 4,545 & 0.06 & 16.50 & 13.34 & 24,735 & 13.10 & 11.48 & 59.28 & 10.34 \\
\hline Thai & 4,384 & 0.05 & 24.07 & 15.94 & 28,581 & 43.73 & 6.73 & 77.28 & 10.58 \\
\hline Other Asians & 22,412 & 0.26 & 14.81 & 14.74 & 35,064 & 45.45 & 8.04 & 58.00 & 6.16 \\
\hline Native Hawaiian & 4,277 & 0.05 & 15.26 & 12.31 & 31,095 & 16.86 & 10.44 & 1.82 & 14.99 \\
\hline Samoan & 2,101 & 0.03 & 21.42 & 15.75 & 22,731 & 11.32 & 14.28 & 13.82 & 8.19 \\
\hline Guamanian & 1,612 & 0.02 & 14.46 & 10.72 & 33,138 & 18.09 & 9.23 & 1.52 & 12.40 \\
\hline Other Pacific Islanders & 1,736 & 0.02 & 17.53 & 15.23 & 25,191 & 16.03 & 13.59 & 38.29 & 8.33 \\
\hline All other groups ${ }^{4}$ & 180,530 & 2.09 & 19.96 & 15.56 & 30,345 & 28.01 & 11.88 & 9.96 & 14.82 \\
\hline
\end{tabular}

${ }^{1}$ Children under 18 years of age. ${ }^{2}$ Population aged 18 years and older. ${ }^{3}$ Population aged 25 years and older. ${ }^{4}$ This category includes multiple race groups. AN: Alaska Native.

or shopping due to a physical, mental, or emotional condition $[18,19,36]$.

For children under 5 years old, hearing and vision difficulties were used to determine disability status. For children aged 5-14, disability status was determined from hearing, vision, cognitive, ambulatory, and self-care difficulties. For people aged $\geq 15$ years, an individual was considered to have a disability if s/he had difficulty with any one of the 6 disability types [36]. The other dependent variable, health insurance coverage, was also dichotomous. A respondent was considered to have health insurance if $s /$ he reported having any type of private health insurance or public insurance such as Medicaid, Medicare, TRICARE, VA, or Indian Health Service insurance plan [36].

2.2. Independent Variables. Race/ethnicity was classified into 26 categories as shown in Tables 1-3 and included all of the major racial/ethnic groups such as non-Hispanic
Whites, Blacks, American Indians/Alaska Natives, Mexicans, Central/South Americans, Puerto Ricans, Cubans, Asian Indians, Chinese, Filipinos, Japanese, Koreans, Vietnamese, Cambodians, Hawaiians, and some of the newest Asian groups such as Bangladeshis, Pakistanis, Laotians, Thais, and Hmong. With the exception of a residual category of other races that included multiple race groups, all racial/ethnic groups in this study were based on "single race", indicating that people in these groups indicated only one racial identity [36]. Nativity/immigrant status was defined on the basis of individuals' place of birth [6-8, 36]. US-born people were those born in one of the 50 states, Washington, DC, or US territories. Immigrants or foreign-born people refer to those born outside these areas and who were not a US citizen at birth $[6-8,36]$. The joint variable of ethnic immigrant status included 48 categories, with most of the racial/ethnic groups divided into the US-born and foreign-born categories (Tables 4 and 5). Note that American Indians/Alaska Natives, 
TABLE 2: Prevalence, unadjusted, and adjusted odds of disability and lack of health insurance among US children under 18 years of age according to racial/ethnic, socioeconomic, and demographic characteristics: the 2008-2010 American Community Survey $(N=2,079,138)$.

\begin{tabular}{|c|c|c|c|c|c|c|c|c|c|c|c|c|}
\hline \multirow{3}{*}{$\begin{array}{l}\text { Racial/ethnic and } \\
\text { socioeconomic groups }\end{array}$} & \multicolumn{6}{|c|}{ Disability } & \multicolumn{6}{|c|}{ No health insurance } \\
\hline & \multicolumn{2}{|c|}{ Prevalence } & \multicolumn{2}{|c|}{ Unadj.odds ratio } & \multicolumn{2}{|c|}{ Adjusted odds ratio $^{1}$} & \multicolumn{2}{|c|}{ Prevalence } & \multicolumn{2}{|c|}{ Unadj. odds ratio } & \multicolumn{2}{|c|}{ Adjusted odds ratio } \\
\hline & $\%$ & SE & OR & $95 \% \mathrm{CI}$ & OR & $95 \% \mathrm{CI}$ & $\%$ & SE & OR & $95 \% \mathrm{CI}$ & OR & $95 \% \mathrm{CI}$ \\
\hline \multicolumn{13}{|l|}{ Race/ethnicity } \\
\hline Non-Hispanic White & 4.08 & 0.02 & 1.00 & Reference & 1.00 & Reference & 5.89 & 0.02 & 1.00 & Reference & 1.00 & Reference \\
\hline Mexican & 3.28 & 0.03 & 0.87 & $0.85-0.89$ & 0.69 & $0.67-0.71$ & 17.60 & 0.07 & 3.39 & $3.35-3.44$ & 2.12 & $2.09-2.14$ \\
\hline Puerto Rican & 6.82 & 0.13 & 1.78 & $1.71-1.86$ & 1.42 & $1.36-1.48$ & 6.46 & 0.13 & 1.11 & $1.06-1.16$ & 0.87 & $0.83-0.91$ \\
\hline Cuban & 3.39 & 0.19 & 0.89 & $0.80-0.99$ & 0.89 & $0.80-1.00$ & 11.71 & 0.30 & 1.93 & $1.80-2.06$ & 1.35 & $1.26-1.45$ \\
\hline Central/South American & 2.88 & 0.07 & 0.77 & $0.74-0.81$ & 0.70 & $0.66-0.73$ & 16.29 & 0.15 & 3.09 & $3.01-3.16$ & 1.81 & $1.76-1.86$ \\
\hline Other Hispanics & 4.63 & 0.15 & 1.20 & $1.12-1.28$ & 1.02 & $0.96-1.09$ & 9.57 & 0.20 & 1.81 & $1.73-1.90$ & 1.43 & $1.37-1.51$ \\
\hline Non-Hispanic Black & 4.94 & 0.05 & 1.44 & $1.41-1.47$ & 1.04 & $1.02-1.06$ & 7.95 & 0.06 & 1.47 & $1.44-1.49$ & 1.01 & $1.00-1.03$ \\
\hline American Indian/AN & 5.71 & 0.17 & 1.41 & $1.33-1.51$ & 1.03 & $0.97-1.10$ & 21.86 & 0.30 & 4.74 & $4.58-4.92$ & 3.51 & $3.38-3.64$ \\
\hline Asian Indian & 1.51 & 0.09 & 0.39 & $0.35-0.44$ & 0.57 & $0.51-0.64$ & 6.54 & 0.17 & 1.04 & $0.97-1.10$ & 0.94 & $0.88-1.01$ \\
\hline Chinese & 1.53 & 0.09 & 0.41 & $0.37-0.46$ & 0.49 & $0.44-0.55$ & 6.19 & 0.16 & 1.02 & $0.96-1.08$ & 0.77 & $0.72-0.82$ \\
\hline Filipino & 1.98 & 0.12 & 0.51 & $0.45-0.57$ & 0.59 & $0.52-0.66$ & 5.79 & 0.20 & 1.06 & $0.99-1.14$ & 0.72 & $0.67-0.78$ \\
\hline Japanese & 1.43 & 0.26 & 0.41 & $0.30-0.56$ & 0.52 & $0.38-0.71$ & 4.12 & 0.38 & 0.68 & $0.55-0.84$ & 0.54 & $0.44-0.67$ \\
\hline Korean & 1.87 & 0.16 & 0.48 & $0.41-0.56$ & 0.52 & $0.44-0.62$ & 17.54 & 0.41 & 3.22 & $3.02-3.42$ & 1.87 & $1.75-2.00$ \\
\hline Vietnamese & 1.88 & 0.14 & 0.53 & $0.46-0.60$ & 0.50 & $0.44-0.57$ & 10.24 & 0.28 & 1.77 & $1.66-1.89$ & 1.13 & $1.06-1.22$ \\
\hline Cambodian & 3.12 & 0.49 & 0.97 & $0.75-1.25$ & 0.75 & $0.58-0.98$ & 10.66 & 0.73 & 1.77 & $1.49-2.10$ & 1.08 & $0.90-1.29$ \\
\hline Bangladeshi & 1.96 & 0.52 & 0.53 & $0.33-0.86$ & 0.49 & $0.30-0.80$ & 12.18 & 1.15 & 2.33 & $1.87-2.89$ & 1.08 & $0.86-1.35$ \\
\hline Pakistani & 1.63 & 0.27 & 0.46 & $0.35-0.62$ & 0.46 & $0.35-0.61$ & 12.83 & 0.61 & 2.17 & $1.92-2.45$ & 1.25 & $1.10-1.42$ \\
\hline Hmong & 2.77 & 0.35 & 0.67 & $0.52-0.88$ & 0.49 & $0.38-0.64$ & 5.81 & 0.53 & 1.19 & $1.00-1.42$ & 0.57 & $0.47-0.68$ \\
\hline Laotian & 3.04 & 0.52 & 0.82 & $0.60-1.13$ & 0.65 & $0.47-0.90$ & 12.19 & 0.90 & 2.11 & $1.75-2.54$ & 1.43 & $1.18-1.73$ \\
\hline Thai & 2.02 & 0.59 & 0.59 & $0.36-0.98$ & 0.57 & $0.34-0.93$ & 13.00 & 1.29 & 2.54 & $2.02-3.19$ & 1.22 & $0.95-1.55$ \\
\hline Other Asians & 1.95 & 0.17 & 0.52 & $0.44-0.61$ & 0.60 & $0.50-0.70$ & 7.48 & 0.31 & 1.29 & $1.17-1.42$ & 0.90 & $0.81-0.99$ \\
\hline Native Hawaiian & 3.26 & 0.58 & 0.85 & $0.60-1.20$ & 0.70 & $0.49-0.99$ & 4.22 & 0.64 & 0.76 & $0.55-1.04$ & 0.61 & $0.45-0.84$ \\
\hline Samoan & 1.93 & 0.62 & 0.64 & $0.39-1.03$ & 0.45 & $0.28-0.73$ & 6.44 & 0.93 & 1.13 & $0.82-1.56$ & 0.76 & $0.55-1.05$ \\
\hline Guamanian & 2.38 & 0.91 & 0.76 & $0.42-1.38$ & 0.64 & $0.35-1.17$ & 8.03 & 1.43 & 1.54 & $1.05-2.25$ & 1.29 & $0.88-1.88$ \\
\hline Other Pacific Islanders & 1.29 & 0.55 & 0.43 & $0.23-0.81$ & 0.36 & $0.19-0.67$ & 12.33 & 1.29 & 2.07 & $1.59-2.71$ & 1.43 & $1.09-1.89$ \\
\hline All other groups ${ }^{2}$ & 5.00 & 0.08 & 1.28 & $1.24-1.32$ & 1.25 & $1.21-1.30$ & 6.42 & 0.08 & 1.13 & $1.10-1.16$ & 1.02 & $0.99-1.05$ \\
\hline \multicolumn{13}{|l|}{$\begin{array}{l}\text { Poverty status (ratio of } \\
\text { family income to poverty } \\
\text { threshold) }\end{array}$} \\
\hline$<100 \%$ & 6.09 & 0.04 & 3.12 & $3.05-3.20$ & 3.62 & $3.53-3.72$ & 12.37 & 0.05 & 6.85 & $6.68-7.02$ & 5.50 & $5.36-5.64$ \\
\hline $100-199 \%$ & 4.67 & 0.03 & 2.29 & $2.23-2.35$ & 2.54 & $2.47-2.60$ & 13.73 & 0.05 & 7.54 & $7.36-7.72$ & 6.24 & $6.09-6.40$ \\
\hline $200-299 \%$ & 3.64 & 0.03 & 1.72 & $1.67-1.76$ & 1.79 & $1.75-1.84$ & 9.65 & 0.05 & 5.01 & $4.89-5.14$ & 4.57 & $4.46-4.69$ \\
\hline $300-399 \%$ & 3.11 & 0.03 & 1.43 & $1.39-1.47$ & 1.45 & $1.41-1.50$ & 5.62 & 0.04 & 2.75 & $2.67-2.82$ & 2.65 & $2.58-2.72$ \\
\hline $400-499 \%$ & 2.71 & 0.04 & 1.24 & $1.20-1.28$ & 1.25 & $1.20-1.29$ & 3.59 & 0.04 & 1.67 & $1.62-1.73$ & 1.65 & $1.60-1.71$ \\
\hline$\geq 500 \%$ & 2.20 & 0.02 & 1.00 & Reference & 1.00 & Reference & 2.14 & 0.02 & 1.00 & Reference & 1.00 & reference \\
\hline Unknown & 10.83 & 0.19 & 6.34 & $6.09-6.59$ & 6.56 & $6.30-6.84$ & 12.39 & 0.18 & 6.86 & $6.58-7.15$ & 6.05 & $5.80-6.31$ \\
\hline \multicolumn{13}{|l|}{ Age (years) } \\
\hline $0-5$ & 1.24 & 0.01 & 1.00 & Reference & 1.00 & Reference & 8.45 & 0.03 & 1.00 & Reference & 1.00 & Reference \\
\hline $6-11$ & 5.20 & 0.03 & 4.48 & $4.37-4.59$ & 4.69 & $4.57-4.81$ & 10.47 & 0.03 & 1.20 & $1.18-1.21$ & 1.18 & $1.17-1.20$ \\
\hline $12-17$ & 5.60 & 0.03 & 4.91 & $4.79-5.03$ & 5.33 & $5.20-5.47$ & 7.08 & 0.03 & 1.53 & $1.51-1.55$ & 1.54 & $1.52-1.56$ \\
\hline \multicolumn{13}{|l|}{ Gender } \\
\hline Male & 5.00 & 0.02 & 1.67 & $1.65-1.70$ & 1.68 & $1.66-1.70$ & 8.70 & 0.03 & 1.00 & 0.99-1.01 & 1.00 & $0.99-1.01$ \\
\hline Female & 3.05 & 0.02 & 1.00 & Reference & 1.00 & Reference & 8.70 & 0.03 & 1.00 & Reference & 1.00 & Reference \\
\hline
\end{tabular}


TABLE 2: Continued.

\begin{tabular}{|c|c|c|c|c|c|c|c|c|c|c|c|c|}
\hline \multirow{3}{*}{$\begin{array}{l}\text { Racial/ethnic and } \\
\text { socioeconomic groups }\end{array}$} & \multicolumn{6}{|c|}{ Disability } & \multicolumn{6}{|c|}{ No health insurance } \\
\hline & \multicolumn{2}{|c|}{ Prevalence } & \multicolumn{2}{|c|}{ Unadj.odds ratio } & \multicolumn{2}{|c|}{ Adjusted odds ratio ${ }^{1}$} & \multicolumn{2}{|c|}{ Prevalence } & \multicolumn{2}{|c|}{ Unadj. odds ratio } & \multicolumn{2}{|c|}{ Adjusted odds ratio ${ }^{1}$} \\
\hline & $\%$ & SE & OR & $95 \% \mathrm{CI}$ & OR & $95 \% \mathrm{CI}$ & $\%$ & SE & OR & $95 \% \mathrm{CI}$ & OR & $95 \% \mathrm{CI}$ \\
\hline \multicolumn{13}{|l|}{ Immigrant status } \\
\hline Immigrant & 2.78 & 0.06 & 0.74 & $0.71-0.78$ & 0.66 & $0.63-0.69$ & 32.16 & 0.17 & 5.21 & $5.12-5.30$ & 3.66 & $3.59-3.74$ \\
\hline US-born & 4.10 & 0.01 & 1.00 & Reference & 1.00 & Reference & 7.76 & 0.02 & 1.00 & Reference & 1.00 & Reference \\
\hline
\end{tabular}

OR: odds ratio; SE: standard error; CI: confidence interval; AN: Alaska Native. ${ }^{1}$ Adjusted by logistic regression model for age, gender, race/ethnicity, immigrant status, and poverty status. ${ }^{2}$ This category includes multiple race groups.

Hawaiians, Samoans, and Guamanians are considered nativeborn, although a small percentage of people in these groups may have been born outside the USA [36].

Using the social determinants of health framework and past research as a guide, we considered, in addition to race/ethnicity and nativity/immigrant status, the following socioeconomic and demographic covariates that are known to be associated with disability and health insurance: age, gender, marital status, and three measures of socioeconomic status (SES): educational attainment, poverty status measured as a ratio of family income to the poverty threshold, and employment status [11-17, 31, 32, 39]. These covariates were measured as shown in Tables 1-3.

2.3. Statistical Methods. Multivariate logistic regression was used to model the association between ethnicity and socioeconomic factors and the binary outcomes of disability and health insurance $[40,41]$. The two-sample $t$ test was used to test the difference in prevalence between any two groups. Additionally, we used root-mean-square-deviation (RMSD) as a summary measure of ethnic disparities in disability and health insurance coverage [42]. The RMSD is similar to the square root of the variance, except that the average squared deviations are calculated using a "standard" estimate other than the sample mean. The RMSD is given by the formula

$$
\operatorname{RMSD}=\operatorname{SQRT}\left\{\sum_{i} \frac{\left(X_{r i}-X_{r l}\right)^{2}}{I}\right\},
$$

where $X_{r i}$ is the disability or uninsurance rate for the $i$ th group $(i=1,2, \ldots, 26), X_{r l}$ is the corresponding statistic for the "standard" group (total US population) or group with the lowest rate of disability or uninsurance (i.e., Japanese children or Asian Indian adults), and $I$ is the number of ethnic groups (26) being compared.

While RMSD is a measure of absolute health disparity, the coefficient of variation (CV) of the RMSD provides an estimate of relative disparity and is given by

$$
\mathrm{CV}(\mathrm{RMSD})=\left(\frac{\mathrm{RMSD}}{X_{r l}}\right) \times 100 ; \quad X_{r l}>0 .
$$

\section{Results}

3.1. Socioeconomic and Demographic Profiles of Racial/Ethnic Groups. Racial/ethnic groups in the USA vary substantially in their socioeconomic characteristics (Table 1). While NonHispanic Whites and the major Asian-American groups such as Asian Indians, Chinese, Filipinos, Japanese, and Koreans had higher education and income levels and lower poverty and unemployment rates, Blacks, American Indians/Alaska Natives, Native Hawaiians, Samoans, Mexican, Puerto Ricans, Central and South Americans, Cambodians, Hmong, and Laotians had substantially lower SES levels. Approximately one third of Black, American Indian/Alaska Native, Hmong, Mexican, and Puerto Rican children were below the poverty line, compared with $5.1 \%$ of Filipinos and $6.4 \%$ of Japanese children. Approximately $24 \%$ of Hmong and American Indian/Alaska Native adults were below the poverty line, compared with $5.6 \%$ of Filipino adults. Only $9.3 \%$ of Mexicans were college graduates, compared with $71.3 \%$ of Asian Indians. More than two thirds of the Asian Indian, Chinese, Filipino, Korean, Vietnamese, Bangladeshi, Pakistani, and Thai populations in the USA were foreignborn, compared with $3.8 \%$ of Whites and $7.9 \%$ of Blacks.

3.2. Social Inequalities in Disability. During 2008-2010, $12.5 \%$, or 38.4 million people in the US, had a disability. While $4 \%$ or 3.0 million children under 18 years of age had a disability, $15.2 \%$ or 35.4 million adults had a disability. Disability rates varied from a low of $1.4 \%$ for Japanese children and $1.5 \%$ for Asian Indian and Chinese children to a high of 5.7\% for American Indian/Alaska Native children and $6.8 \%$ for Puerto Rican children (Table 2 and Figure 1). The prevalence of disability in adults ranged from $5.6 \%$ among Asian Indians to $17.9 \%$ among Blacks and $22.0 \%$ among American Indians/Alaska Natives (Table 3 and Figure 1). After adjusting for socioeconomic differences, children in almost all Asian and Hispanic subgroups had a significantly lower risk of disability and Puerto Rican children had $42 \%$ higher odds of disability than White children (Table 2). While Chinese, Koreans, Japanese, Vietnamese, Asian Indian, Thai, Mexican, and Central/South American adults had lower adjusted odds of disability than Whites, American Indian/Alaska Native adults had 32\% higher adjusted odds and Filipino, Cambodian, and Cuban adults 11-12\% higher odds than Whites (Table 3). Socioeconomic gradients in disability were marked among both children and adults, with those below the poverty line having 2.2-3.6 times higher odds of disability than their affluent counterparts (Tables 2 and 3 and Figure 2). Adults with less than a high school education had 2.7 times higher adjusted odds of disability 
TABLE 3: Prevalence, unadjusted, and adjusted odds of disability and lack of health insurance among US adults aged 18+ years according to racial/ethnic, socioeconomic, and demographic characteristics: the 2008-2010 American Community Survey $(N=7,013,939)$.

\begin{tabular}{|c|c|c|c|c|c|c|c|c|c|c|c|c|}
\hline \multirow{3}{*}{$\begin{array}{l}\text { Racial/ethnic and } \\
\text { socioeconomic groups }\end{array}$} & \multicolumn{6}{|c|}{ Disability } & \multicolumn{6}{|c|}{ No health insurance } \\
\hline & \multicolumn{2}{|c|}{ Prevalence } & \multicolumn{2}{|c|}{$\begin{array}{l}\text { Unadjusted odds } \\
\text { ratio }\end{array}$} & \multicolumn{2}{|c|}{$\begin{array}{l}\text { Adjusted } \\
\text { odds ratio }^{1}\end{array}$} & \multicolumn{2}{|c|}{ Prevalence } & \multicolumn{2}{|c|}{$\begin{array}{l}\text { Unadjusted odds } \\
\text { ratio }\end{array}$} & \multicolumn{2}{|c|}{$\begin{array}{l}\text { Adjusted odds } \\
\text { ratio }^{1}\end{array}$} \\
\hline & $\%$ & SE & OR & $95 \%$ CI & OR & $95 \% \mathrm{CI}$ & $\%$ & SE & OR & $95 \% \mathrm{CI}$ & OR & $95 \% \mathrm{CI}$ \\
\hline \multicolumn{13}{|l|}{ Race/ethnicity } \\
\hline Non-Hispanic White & 16.05 & 0.02 & 1.00 & Reference & 1.00 & Reference & 11.82 & 0.01 & 1.00 & Reference & 1.00 & Reference \\
\hline Mexican & 10.13 & 0.05 & 0.65 & $0.65-0.66$ & 0.83 & $0.82-0.84$ & 43.61 & 0.07 & 5.66 & $5.63-5.70$ & 1.87 & $1.85-1.89$ \\
\hline Puerto Rican & 17.52 & 0.14 & 1.15 & $1.13-1.17$ & 1.06 & $1.04-1.09$ & 19.41 & 0.14 & 1.92 & $1.89-1.96$ & 0.92 & $0.91-0.94$ \\
\hline Cuban & 15.48 & 0.19 & 1.00 & $0.97-1.03$ & 1.12 & $1.08-1.15$ & 27.10 & 0.22 & 2.59 & $2.53-2.66$ & 1.46 & $1.42-1.51$ \\
\hline Central/South American & 7.89 & 0.08 & 0.49 & $0.48-0.49$ & 0.85 & $0.83-0.87$ & 41.35 & 0.13 & 5.11 & $5.05-5.17$ & 1.65 & $1.63-1.68$ \\
\hline Other Hispanics & 16.65 & 0.18 & 1.11 & $1.09-1.14$ & 1.17 & $1.13-1.20$ & 21.80 & 0.19 & 2.14 & $2.09-2.19$ & 1.25 & $1.22-1.28$ \\
\hline Non-Hispanic Black & 17.91 & 0.05 & 1.28 & $1.27-1.29$ & 1.02 & $1.02-1.03$ & 22.72 & 0.05 & 2.34 & $2.32-2.35$ & 1.11 & $1.10-1.12$ \\
\hline American Indian/AN & 22.03 & 0.19 & 1.56 & $1.53-1.59$ & 1.32 & $1.29-1.36$ & 32.73 & 0.21 & 3.92 & $3.84-4.00$ & 2.20 & $2.15-2.24$ \\
\hline Asian Indian & 5.57 & 0.10 & 0.31 & $0.30-0.32$ & 0.94 & $0.90-0.98$ & 13.46 & 0.14 & 1.20 & $1.17-1.24$ & 0.86 & $0.84-0.89$ \\
\hline Chinese & 6.97 & 0.09 & 0.39 & $0.38-0.41$ & 0.66 & $0.64-0.68$ & 15.15 & 0.12 & 1.37 & $1.34-1.40$ & 0.80 & $0.78-0.82$ \\
\hline Filipino & 9.17 & 0.12 & 0.52 & $0.51-0.53$ & 1.16 & $1.13-1.20$ & 11.95 & 0.13 & 1.07 & $1.04-1.10$ & 0.77 & $0.75-0.79$ \\
\hline Japanese & 11.66 & 0.23 & 0.75 & $0.72-0.78$ & 0.80 & $0.77-0.84$ & 7.29 & 0.16 & 0.56 & $0.53-0.60$ & 0.63 & $0.59-0.67$ \\
\hline Korean & 6.54 & 0.15 & 0.38 & $0.37-0.40$ & 0.67 & $0.64-0.71$ & 26.75 & 0.25 & 2.83 & $2.76-2.91$ & 1.85 & $1.80-1.91$ \\
\hline Vietnamese & 9.58 & 0.17 & 0.57 & $0.55-0.59$ & 0.92 & $0.88-0.95$ & 22.73 & 0.23 & 2.30 & $2.24-2.37$ & 0.90 & $0.87-0.93$ \\
\hline Cambodian & 13.03 & 0.52 & 0.76 & $0.70-0.83$ & 1.11 & $1.01-1.23$ & 25.33 & 0.65 & 2.70 & $2.51-2.89$ & 0.76 & $0.70-0.82$ \\
\hline Bangladeshi & 6.61 & 0.60 & 0.35 & $0.29-0.42$ & 0.86 & $0.70-1.05$ & 27.26 & 1.04 & 2.98 & $2.67-3.32$ & 1.11 & $0.99-1.25$ \\
\hline Pakistani & 6.75 & 0.33 & 0.36 & $0.33-0.40$ & 0.91 & $0.82-1.02$ & 29.33 & 0.58 & 3.13 & $2.95-3.32$ & 1.54 & $1.44-1.65$ \\
\hline Hmong & 10.84 & 0.59 & 0.64 & $0.57-0.71$ & 1.06 & $0.93-1.20$ & 21.26 & 0.77 & 2.39 & $2.18-2.61$ & 0.56 & $0.51-0.62$ \\
\hline Laotian & 10.75 & 0.56 & 0.68 & $0.61-0.75$ & 1.07 & $0.96-1.20$ & 20.98 & 0.68 & 2.15 & $1.98-2.35$ & 0.65 & $0.59-0.71$ \\
\hline Thai & 6.28 & 0.41 & 0.36 & $0.31-0.41$ & 0.70 & $0.61-0.80$ & 23.96 & 0.67 & 2.41 & $2.23-2.61$ & 1.21 & $1.11-1.32$ \\
\hline Other Asians & 6.78 & 0.21 & 0.38 & $0.36-0.41$ & 0.82 & $0.76-0.87$ & 19.14 & 0.30 & 1.81 & $1.74-1.89$ & 0.82 & $0.78-0.86$ \\
\hline Native Hawaiian & 15.17 & 0.65 & 1.01 & $0.92-1.11$ & 1.06 & $0.96-1.18$ & 13.90 & 0.59 & 1.38 & $1.25-1.53$ & 0.88 & $0.79-0.98$ \\
\hline Samoan & 13.49 & 0.91 & 0.81 & $0.70-0.94$ & 1.16 & $0.98-1.36$ & 21.38 & 1.05 & 2.20 & $1.93-2.50$ & 0.86 & $0.75-0.99$ \\
\hline Guamanian & 11.02 & 0.92 & 0.67 & $0.57-0.80$ & 0.99 & $0.82-1.20$ & 19.23 & 1.04 & 1.70 & $1.46-1.97$ & 1.07 & $0.90-1.26$ \\
\hline Other Pacific Islanders & 10.70 & 0.91 & 0.60 & $0.50-0.73$ & 0.93 & $0.76-1.14$ & 24.83 & 1.23 & 2.60 & $2.27-2.98$ & 0.98 & $0.84-1.13$ \\
\hline All other groups ${ }^{2}$ & 17.38 & .13 & 1.18 & $1.16-1.20$ & 1.65 & $1.62-1.68$ & 21.04 & 0.12 & 2.09 & $2.05-2.12$ & 1.17 & $1.15-1.19$ \\
\hline \multicolumn{13}{|l|}{$\begin{array}{l}\text { Education (years of school } \\
\text { completed) }\end{array}$} \\
\hline $0-11$ & 28.62 & 0.05 & 5.53 & $5.49-5.57$ & 2.74 & $2.72-2.77$ & 32.28 & 27.07 & 6.26 & $6.21-6.31$ & 2.99 & $2.96-3.02$ \\
\hline 12 & 18.31 & 0.03 & 2.90 & $2.88-2.91$ & 1.74 & $1.73-1.76$ & 21.13 & 17.58 & 3.60 & $3.57-3.63$ & 2.41 & $2.39-2.43$ \\
\hline $13-15$ & 12.23 & 0.02 & 1.78 & $1.77-1.80$ & 1.53 & $1.52-1.54$ & 15.59 & 13.33 & 2.59 & $2.57-2.61$ & 1.66 & $1.64-1.67$ \\
\hline$\geq 16$ & 7.46 & 0.02 & 1.00 & Reference & 1.00 & Reference & 6.76 & 5.60 & 1.00 & Reference & 1.00 & Reference \\
\hline \multicolumn{13}{|l|}{$\begin{array}{l}\text { Poverty status (ratio of } \\
\text { family income to poverty } \\
\text { threshold) }\end{array}$} \\
\hline$<100 \%$ & 24.30 & 0.05 & 4.08 & $4.05-4.11$ & 2.27 & $2.25-2.29$ & 35.85 & 0.05 & 12.13 & $12.03-12.24$ & 5.62 & $5.56-5.67$ \\
\hline $100-199 \%$ & 21.86 & 0.04 & 3.57 & $3.54-3.59$ & 1.91 & $1.90-1.93$ & 29.97 & 0.04 & 8.62 & $8.55-8.69$ & 5.46 & $5.41-5.51$ \\
\hline $200-299 \%$ & 15.78 & 0.04 & 2.32 & $2.30-2.33$ & 1.50 & $1.49-1.51$ & 20.04 & 0.04 & 5.04 & $5.00-5.08$ & 3.41 & $3.38-3.44$ \\
\hline $300-399 \%$ & 12.69 & 0.03 & 1.75 & $1.74-1.76$ & 1.33 & $1.32-1.34$ & 12.94 & 0.03 & 2.98 & $2.95-3.01$ & 2.18 & $2.16-2.20$ \\
\hline $400-499 \%$ & 10.62 & 0.04 & 1.40 & $1.39-1.41$ & 1.21 & $1.20-1.22$ & 8.92 & 0.03 & 1.96 & $1.94-1.98$ & 1.55 & $1.54-1.57$ \\
\hline$\geq 500 \%$ & 8.05 & 0.02 & 1.00 & Reference & 1.00 & Reference & 4.76 & 0.01 & 1.00 & Reference & 1.00 & Reference \\
\hline Unknown & 32.93 & 0.11 & 7.17 & $7.09-7.24$ & 3.27 & $3.23-3.31$ & 26.84 & 0.10 & 10.53 & $10.41-10.65$ & 5.91 & $5.83-6.00$ \\
\hline \multicolumn{13}{|l|}{ Employment status } \\
\hline Unemployed & 11.00 & 0.05 & 2.10 & $2.08-2.12$ & 1.68 & $1.66-1.70$ & 46.18 & 0.08 & 4.72 & $4.68-4.75$ & 2.88 & $2.86-2.91$ \\
\hline Not in labor force & 33.41 & 0.03 & 8.45 & $8.41-8.49$ & 4.10 & $4.08-4.12$ & 14.17 & 0.02 & 0.84 & $0.84-0.84$ & 1.00 & $1.00-1.01$ \\
\hline Employed & 5.59 & 0.01 & 1.00 & Reference & 1.00 & Reference & 16.48 & 0.02 & 1.00 & Reference & 1.00 & Reference \\
\hline
\end{tabular}


TABLE 3: Continued.

\begin{tabular}{|c|c|c|c|c|c|c|c|c|c|c|c|c|}
\hline \multirow{3}{*}{$\begin{array}{l}\text { Racial/ethnic and } \\
\text { socioeconomic groups }\end{array}$} & \multicolumn{6}{|c|}{ Disability } & \multicolumn{6}{|c|}{ No health insurance } \\
\hline & \multicolumn{2}{|c|}{ Prevalence } & \multicolumn{2}{|c|}{$\begin{array}{l}\text { Unadjusted odds } \\
\text { ratio }\end{array}$} & \multicolumn{2}{|c|}{$\begin{array}{l}\text { Adjusted } \\
\text { odds ratio }^{1}\end{array}$} & \multicolumn{2}{|c|}{ Prevalence } & \multicolumn{2}{|c|}{$\begin{array}{l}\text { Unadjusted odds } \\
\text { ratio }\end{array}$} & \multicolumn{2}{|c|}{$\begin{array}{l}\text { Adjusted odds } \\
\text { ratio }^{1}\end{array}$} \\
\hline & $\%$ & SE & OR & $95 \% \mathrm{CI}$ & OR & $95 \% \mathrm{CI}$ & $\%$ & SE & OR & $95 \%$ CI & OR & $95 \% \mathrm{CI}$ \\
\hline \multicolumn{13}{|l|}{ Age (years) } \\
\hline $18-24$ & 5.58 & 0.03 & 1.00 & Reference & 1.00 & Reference & 29.31 & 0.05 & 3.24 & $3.22-3.27$ & 1.43 & $1.42-1.45$ \\
\hline $25-34$ & 5.81 & 0.02 & 1.00 & $0.99-1.01$ & 1.98 & $1.95-2.00$ & 27.48 & 0.04 & 2.79 & $2.77-2.81$ & 2.01 & $1.99-2.02$ \\
\hline $35-44$ & 7.91 & 0.02 & 1.35 & $1.33-1.37$ & 3.28 & $3.24-3.33$ & 20.48 & 0.04 & 1.87 & $1.85-1.88$ & 1.52 & $1.50-1.53$ \\
\hline $45-54$ & 12.81 & 0.03 & 2.28 & $2.26-2.31$ & 5.83 & $5.75-5.90$ & 16.35 & 0.03 & 1.45 & $1.44-1.46$ & 1.34 & $1.33-1.36$ \\
\hline $55-64$ & 19.06 & 0.04 & 3.67 & $3.63-3.71$ & 7.75 & $7.65-7.85$ & 11.80 & 0.03 & 1.00 & Reference & 1.00 & Reference \\
\hline$\geq 65$ & 39.20 & 0.04 & 10.13 & $\begin{array}{c}10.03- \\
10.24\end{array}$ & 9.64 & $9.52-9.77$ & 0.95 & 0.01 & 0.06 & $0.06-0.06$ & 0.04 & 0.04-0.04 \\
\hline \multicolumn{13}{|l|}{ Gender } \\
\hline Male & 14.56 & 0.02 & 0.93 & $0.92-0.93$ & 1.27 & $1.27-1.28$ & 20.03 & 0.02 & 1.41 & $1.40-1.42$ & 1.37 & $1.36-1.38$ \\
\hline Female & 15.82 & 0.02 & 1.00 & Reference & 1.00 & Reference & 14.96 & 0.02 & 1.00 & Reference & 1.00 & Reference \\
\hline \multicolumn{13}{|l|}{ Immigrant status } \\
\hline Immigrant & 9.55 & 0.03 & 0.58 & $0.57-0.58$ & 0.60 & $0.60-0.61$ & 33.61 & 0.05 & 2.85 & $2.84-2.86$ & 2.15 & $2.13-2.16$ \\
\hline US-born & 16.26 & 0.02 & 1.00 & Reference & 1.00 & Reference & 14.40 & 0.01 & 1.00 & Reference & 1.00 & Reference \\
\hline \multicolumn{13}{|l|}{ Marital status } \\
\hline Married & 12.05 & 0.02 & 1.00 & Reference & 1.00 & Reference & 11.40 & 0.01 & 1.00 & Reference & 1.00 & Reference \\
\hline Widowed & 47.25 & 0.07 & 6.08 & $6.04-6.12$ & 1.91 & $1.90-1.93$ & 5.71 & 0.03 & 0.46 & $0.45-0.47$ & 1.56 & $1.53-1.58$ \\
\hline Divorced/separated & 20.97 & 0.04 & 1.88 & $1.87-1.89$ & 1.56 & $1.55-1.57$ & 22.29 & 0.04 & 2.46 & $2.44-2.47$ & 1.93 & $1.91-1.94$ \\
\hline Never married & 10.94 & 0.03 & 0.91 & $0.90-0.91$ & 1.66 & $1.65-1.68$ & 29.09 & 0.03 & 3.60 & $3.58-3.62$ & 1.88 & $1.86-1.89$ \\
\hline
\end{tabular}

OR: odds ratio; SE: standard error; CI: confidence interval; AN: Alaska Native.

${ }^{1}$ Adjusted by logistic regression model for age, gender, race/ethnicity, immigrant status, marital status, education, poverty, and employment status.

${ }^{2}$ This category includes multiple race groups.

than college graduates. The unemployed and those outside the labor force had, respectively, 1.6 and 4.1 times higher adjusted odds than those with a job (Table 3). Differences in socioeconomic characteristics accounted for $60.4 \%$ of racial/ethnic variations in child disability and $89.6 \%$ of ethnic inequality in adult disability.

3.3. Social Inequalities in Health Insurance Coverage. During 2008-2010, $15.3 \%$, or 47.0 million people in the USA, were without health insurance coverage. Approximately $8.7 \%$ or 6.4 million children aged $<18$ lacked health insurance, compared with $17.4 \%$ or 40.5 million adults aged $\geq 18$ years. Ethnic disparities in health insurance coverage were at least as pronounced as those in disability. More than $17 \%$ of Korean, Mexican, and American Indian/Alaska Native children lacked health insurance, compared with $4.1 \%$ of Japanese children and $5.9 \%$ of White children (Table 2). Among adults, Mexicans (43.6\%), Central/South Americans (41.4\%), American Indians/Alaska Natives (32.7\%), Pakistanis $(29.3 \%)$, and Bangladeshis (27.3\%) had the highest health uninsurance rates (Table 3 and Figure 1). After adjusting for socioeconomic differences, American Indian/Alaska Native, Mexican, Korean, Central/South American, and Laotian children had 3.5, 2.1, 1.9, 1.8, and 1.4 times higher odds of lacking health insurance coverage than White children, respectively (Table 2). After adjusting for socioeconomic characteristics, American Indian/Alaska Native, Mexican,
Korean, Central/South American, and Pakistani adults had $2.2,1.9,1.9,1.7$, and 1.5 times higher odds of lacking health insurance coverage than White adults, respectively (Table 3 ).

Socioeconomic gradients in health insurance coverage among children as well as adults were quite steep, with those below the poverty line having 5-6 times higher adjusted odds of uninsurance than their affluent counterparts. Independent of income levels, adults with less than high school education or without a job had almost 3 times higher odds of lacking health insurance coverage than those with a college degree or a job (Table 3). Socioeconomic differences accounted for $24.4 \%$ and $75.1 \%$ of racial/ethnic disparities in health insurance coverage among children and adults, respectively.

3.4. Ethnic-Nativity Disparities in Disability and Health Insurance. Ethnic nativity disparities in disability and health insurance coverage were greater than those by race/ethnicity alone (Tables 4 and 5). Although, overall, immigrants had considerably lower disability rates and higher uninsurance rates (Tables 2 and 3), ethnic nativity patterns show the extent of inequalities by immigrant status. While Black, White, and Mexican immigrant children and adults had lower disability rates than their US-born counterparts, immigrant children and adults in most of the Asian subgroups generally had higher disability rates than their US-born counterparts (Tables 4 and 5 and Figure 3). However, children in most ethnic nativity groups, including White and Black immigrant 
TABLE 4: Prevalence and adjusted odds of disability and lack of health insurance among US children under 18 years of age in 48 ethnic immigrant groups: the 2008-2010 American Community Survey $(N=2,079,138)$.

\begin{tabular}{|c|c|c|c|c|c|c|c|c|c|c|}
\hline \multirow{3}{*}{ Ethnic immigrant group } & \multicolumn{5}{|c|}{ Disability } & \multicolumn{5}{|c|}{ No health insurance } \\
\hline & \multicolumn{2}{|c|}{ Prevalence } & \multicolumn{3}{|c|}{ Adjusted odds ratio ${ }^{1}$} & \multicolumn{2}{|c|}{ Prevalence } & \multicolumn{3}{|c|}{ Adjusted odds ratio ${ }^{1}$} \\
\hline & $\%$ & SE & OR & \multicolumn{2}{|c|}{$95 \% \mathrm{CI}$} & $\%$ & SE & OR & \multicolumn{2}{|c|}{$95 \% \mathrm{CI}$} \\
\hline Non-Hispanic White, US-born & 4.09 & 0.02 & 1.00 & \multicolumn{2}{|c|}{ Reference } & 5.81 & 0.02 & 1.00 & \multicolumn{2}{|c|}{ Reference } \\
\hline Non-Hispanic White, immigrant & 3.21 & 0.17 & 0.50 & 0.37 & 0.68 & 13.64 & 0.28 & 1.97 & 1.66 & 2.33 \\
\hline Mexican, US-born & 3.32 & 0.04 & 0.70 & 0.64 & 0.76 & 14.23 & 0.07 & 2.13 & 2.01 & 2.25 \\
\hline Mexican, immigrant & 2.82 & 0.12 & 0.42 & 0.34 & 0.52 & 54.76 & 0.33 & 10.70 & 9.81 & 11.68 \\
\hline Puerto Rican, US-born & 6.82 & 0.13 & 1.25 & 1.07 & 1.47 & 6.44 & 0.13 & 1.02 & 0.87 & 1.20 \\
\hline Puerto Rican, immigrant & 5.41 & 3.32 & 5.07 & 0.91 & 28.20 & 22.82 & 4.48 & 4.24 & 0.78 & 23.21 \\
\hline Cuban, US-born & 3.35 & 0.20 & 0.60 & 0.36 & 0.99 & 9.05 & 0.29 & 1.71 & 1.25 & 2.34 \\
\hline Cuban, immigrant & 3.66 & 0.54 & 0.62 & 0.27 & 1.42 & 28.04 & 1.26 & 3.20 & 2.13 & 4.81 \\
\hline Central/South American, US-born & 2.92 & 0.08 & 0.63 & 0.51 & 0.78 & 12.49 & 0.15 & 2.05 & 1.81 & 2.32 \\
\hline Central/South American, immigrant & 2.69 & 0.18 & 0.37 & 0.25 & 0.53 & 36.04 & 0.49 & 5.61 & 4.89 & 6.43 \\
\hline Other Hispanics, US-born & 4.64 & 0.15 & 1.15 & 0.93 & 1.44 & 9.05 & 0.20 & 1.27 & 1.04 & 1.55 \\
\hline Other Hispanics, immigrant & 4.20 & 0.92 & 0.99 & 0.35 & 2.78 & 30.65 & 1.94 & 3.14 & 1.63 & 6.06 \\
\hline Non-Hispanic Black, US-born & 4.99 & 0.05 & 0.98 & 0.91 & 1.05 & 7.60 & 0.05 & 1.16 & 1.09 & 1.23 \\
\hline Non-Hispanic Black, immigrant & 3.01 & 0.24 & 0.64 & 0.44 & 0.93 & 22.08 & 0.53 & 3.22 & 2.65 & 3.91 \\
\hline American Indian/Alaska Native & 5.71 & 0.17 & 1.07 & 0.87 & 1.32 & 21.86 & 0.30 & 3.56 & 3.11 & 4.07 \\
\hline Asian Indian, US-born & 1.32 & 0.09 & 0.33 & 0.16 & 0.66 & 4.95 & 0.17 & 1.31 & 0.91 & 1.88 \\
\hline Asian Indian, immigrant & 2.21 & 0.26 & 0.34 & 0.15 & 0.77 & 12.65 & 0.52 & 3.24 & 2.38 & 4.40 \\
\hline Chinese, US-born & 1.36 & 0.10 & 0.46 & 0.29 & 0.72 & 5.07 & 0.17 & 0.96 & 0.71 & 1.31 \\
\hline Chinese, immigrant & 2.08 & 0.23 & 0.20 & 0.08 & 0.49 & 9.88 & 0.40 & 2.56 & 1.91 & 3.44 \\
\hline Filipino, US-born & 1.97 & 0.14 & 0.60 & 0.37 & 0.96 & 4.15 & 0.19 & 1.04 & 0.73 & 1.48 \\
\hline Filipino, immigrant & 2.00 & 0.26 & 0.62 & 0.34 & 1.14 & 11.93 & 0.57 & 2.43 & 1.76 & 3.36 \\
\hline Japanese, US-born & 1.29 & 0.28 & 0.52 & 0.16 & 1.65 & 4.12 & 0.42 & 1.57 & 0.75 & 3.26 \\
\hline Japanese, immigrant & 1.83 & 0.58 & $\#$ & & & 4.10 & 0.84 & 0.93 & 0.22 & 3.96 \\
\hline Korean, US-born & 1.74 & 0.19 & 0.63 & 0.34 & 1.16 & 14.21 & 0.47 & 3.65 & 2.70 & 4.93 \\
\hline Korean, immigrant & 2.11 & 0.29 & 0.24 & 0.09 & 0.63 & 23.99 & 0.80 & 5.84 & 4.40 & 7.75 \\
\hline Vietnamese, US-born & 1.77 & 0.15 & 0.49 & 0.29 & 0.82 & 9.03 & 0.28 & 1.76 & 1.34 & 2.33 \\
\hline Vietnamese, immigrant & 2.49 & 0.43 & 0.33 & 0.12 & 0.90 & 17.37 & 0.91 & 2.57 & 1.73 & 3.81 \\
\hline Cambodian, US-born & 3.13 & 0.52 & 0.44 & 0.16 & 1.21 & 9.90 & 0.75 & 1.06 & 0.57 & 1.98 \\
\hline Cambodian, immigrant & 3.07 & 1.42 & 1.60 & 0.20 & 12.82 & 17.61 & 2.63 & 2.99 & 0.62 & 14.51 \\
\hline Bangladeshi, US-born & 2.15 & 0.63 & $\#$ & & & 9.81 & 1.21 & 9.96 & 2.94 & 33.74 \\
\hline Bangladeshi, immigrant & 1.43 & 0.92 & $\#$ & & & 18.46 & 2.66 & 6.24 & 2.21 & 17.61 \\
\hline Pakistani, US-born & 1.51 & 0.30 & 0.63 & 0.20 & 2.02 & 10.39 & 0.64 & 1.70 & 0.83 & 3.48 \\
\hline Pakistani, immigrant & 2.07 & 0.58 & 0.21 & 0.03 & 1.53 & 21.84 & 1.62 & 4.40 & 2.51 & 7.71 \\
\hline Hmong, US-born & 2.55 & 0.36 & 0.31 & 0.10 & 0.99 & 6.12 & 0.60 & 0.61 & 0.28 & 1.31 \\
\hline Hmong, immigrant & 4.20 & 1.24 & 1.44 & 0.43 & 4.85 & 3.87 & 1.02 & 0.35 & 0.05 & 2.58 \\
\hline Laotian, US-born & 3.10 & 0.53 & 1.03 & 0.41 & 2.56 & 11.03 & 0.89 & 1.73 & 0.88 & 3.39 \\
\hline Laotian, immigrant & 1.78 & 2.34 & $\#$ & & & 35.32 & 5.75 & 4.90 & 0.86 & 28.03 \\
\hline Thai, US-born & 2.20 & 0.73 & 0.64 & 0.09 & 4.73 & 10.01 & 1.34 & 2.43 & 0.82 & 7.17 \\
\hline Thai, immigrant & 1.66 & 1.01 & \# & & & 19.02 & 2.74 & 3.17 & 1.34 & 7.47 \\
\hline Other Asians, US-born & 1.88 & 0.19 & 0.48 & 0.23 & 1.03 & 5.27 & 0.29 & 1.16 & 0.72 & 1.89 \\
\hline Other Asians, immigrant & 2.25 & 0.48 & 0.23 & 0.06 & 0.95 & 17.48 & 1.07 & 1.81 & 1.03 & 3.15 \\
\hline Native Hawaiian & 3.26 & 0.58 & 0.20 & 0.03 & 1.45 & 4.22 & 0.64 & 0.89 & 0.36 & 2.23 \\
\hline Samoan & 1.93 & 0.62 & 0.72 & 0.22 & 2.35 & 6.44 & 0.93 & 1.05 & 0.42 & 2.67 \\
\hline Guamanian & 2.38 & 0.91 & 0.83 & 0.11 & 6.33 & 8.03 & 1.44 & 2.46 & 0.69 & 8.75 \\
\hline Other Pacific Islanders, US-born & 1.34 & 0.56 & 1.13 & 0.27 & 4.83 & 12.07 & 1.31 & 1.89 & 0.64 & 5.53 \\
\hline
\end{tabular}


TABLE 4: Continued.

\begin{tabular}{|c|c|c|c|c|c|c|c|c|c|c|}
\hline \multirow{3}{*}{ Ethnic immigrant group } & \multicolumn{5}{|c|}{ Disability } & \multicolumn{5}{|c|}{ No health insurance } \\
\hline & \multicolumn{2}{|c|}{ Prevalence } & \multicolumn{3}{|c|}{ Adjusted odds ratio ${ }^{1}$} & \multicolumn{2}{|c|}{ Prevalence } & \multicolumn{3}{|c|}{ Adjusted odds ratio ${ }^{1}$} \\
\hline & $\%$ & SE & OR & & & $\%$ & SE & OR & & \\
\hline Other Pacific Islanders, immigrant & 0.45 & 2.44 & $\#$ & & & 16.17 & 5.95 & $\#$ & & \\
\hline All other groups, US-born & 5.02 & 0.08 & 1.49 & 1.33 & 1.67 & 6.20 & 0.08 & 1.03 & 0.91 & 1.17 \\
\hline All other groups, immigrant & 4.21 & 0.52 & 0.19 & 0.05 & 0.76 & 17.76 & 0.96 & 2.39 & 1.54 & 3.72 \\
\hline
\end{tabular}

OR: odds ratio; SE: standard error; CI: confidence interval. \#: Insufficient data.

${ }^{1}$ Adjusted by logistic regression model for age, gender, and poverty/family income levels.

children, had significantly lower risk of disability than USborn White children, even after adjusting for income levels (Table 4). After adjusting for socioeconomic factors, White and Black adult immigrants had $32-42 \%$ lower odds of disability and US-born Puerto Ricans and American Indians/Alaska Natives had $7 \%$ and $32 \%$ higher odds of disability than US-born Whites, respectively (Table 5).

Approximately 55\% of Mexican immigrant children, $36.0 \%$ of Central/South American immigrant children, and $35.2 \%$ of Laotian immigrant children lacked health insurance, compared with $4.1 \%$ of US- or foreign-born Japanese children and $5.8 \%$ of US-born White children (Table 4 and Figure 4). Even after adjusting for socioeconomic differences, Mexican, Central/South American, and Korean immigrant children had 6-11 times higher odds of lacking health insurance coverage than US-born White children (Table 4). Among adults, Mexican immigrants (57.3\%), Central/South American immigrants (44.5\%), Pakistani immigrants (30.3\%), USborn Cambodians (35.2\%), US-born Laotians (33.1\%), and American Indians/Alaska Natives (32.7\%) had the highest uninsurance rates (Table 5 and Figure 4). Socioeconomic characteristics reduced ethnic nativity differences in adult health insurance; however, Mexican, Korean, Central/South American, Cuban, and Pakistani immigrants maintained 3.34.5 times higher odds of uninsurance than US-born White adults, respectively (Table 5).

3.5. Social Inequalities in Health Insurance among People with Disabilities. Although, overall, people with disabilities were less likely to be uninsured than those without a disability (10.4\% versus $16.0 \%)$, there were marked ethnic disparities in health insurance coverage among the disabled. More than $20 \%$ of Pakistanis, Bangladeshis, American Indians/Alaska Natives, Mexicans, and Central/South Americans with a disability lacked health insurance, compared with $2.3 \%$ of Japanese and $8.2 \%$ of Whites with disabilities (Table 6). When stratified by nativity status, marked ethnic variations were found in both native- and foreign-born individuals with disabilities (data not shown). For example, $>15 \%$ of US-born Mexicans and Central/South Americans with disabilities and $34.0 \%$ of Mexican immigrants with disabilities were uninsured, compared with $2.0 \%$ of US-born Japanese and $6.4 \%$ of White immigrants with disabilities. Age, immigrant status, and socioeconomic characteristics largely accounted for racial/ethnic differences in uninsurance among people with disabilities. However, even after adjusting for socioeconomic and demographic differences, American
Indians/Alaska Natives, Mexicans, Pakistanis, Central/South Americans, and Asian Indians with disabilities had 1.8, 1.7, 1.6, 1.4, and 1.2 times higher odds of uninsurance than their White counterparts, respectively (Table 6). Strong socioeconomic gradients existed, with people with disabilities in the lowest socioeconomic stratum having 2-4 times higher odds of uninsurance than their affluent counterparts (data not shown).

\section{Discussion}

In this study, we used a large, nationally representative database to examine ethnic and socioeconomic disparities in disability and health insurance in the United States. Because it has a large sample size and is conducted annually, the ACS is an important database for studying and monitoring social inequalities in disability and health insurance coverage in the USA. The new, detailed disability and health insurance statistics for various sociodemographic groups, including those for the newest ethnic immigrant groups, presented herein should serve as the benchmark for setting up national health objectives for various ethnic and immigrant groups in the USA and for conducting further research on the impacts of and factors underlying the disability and health insurance processes.

Our study reveals considerable ethnic, nativity, and socioeconomic disparities in both disability and health insurance. Among children, Puerto Ricans were at the greatest risk of disability. Although children in many of the Asian subgroups, after the socioeconomic adjustment, had fairly similar risks of disability, they were much less likely to experience disability than their White, Black, American Indian/Alaska Native, and Hispanic counterparts. There was greater heterogeneity in adult disability risks among the Asian subgroups, with Filipinos, Cambodians, Laotians, Hmong, Vietnamese, Asian Indians, and Pakistanis experiencing higher disability risks than Chinese and Koreans. Greater social and economic disadvantage of American Indians/Alaska Natives, Puerto Ricans, and Blacks puts them at a high risk of disabilities, but, even after controlling for SES, they remain at a higher disability risk compared to most other groups. These results are consistent with previous studies [11-17].

Among Asians, the Southeast Asian subgroups such as Laotians, Cambodians, Hmong, and Vietnamese are at a higher risk of both child and adult disability, which may partly reflect their immigration circumstances and socioeconomic backgrounds. In contrast to the more affluent Asian 
TABLE 5: Prevalence and adjusted odds of disability and lack of health insurance among US adults aged 18+ years in 48 ethnic immigrant groups: the 2008-2010 American Community Survey $(N=7,013,939)$.

\begin{tabular}{|c|c|c|c|c|c|c|c|c|c|c|}
\hline \multirow{3}{*}{ Ethnic immigrant group } & \multicolumn{5}{|c|}{ Disability } & \multicolumn{5}{|c|}{ No health insurance } \\
\hline & \multicolumn{2}{|c|}{ Prevalence } & \multicolumn{3}{|c|}{ Adjusted odds ratio $^{1}$} & \multicolumn{2}{|c|}{ Prevalence } & \multicolumn{3}{|c|}{ Adjusted odds ratio ${ }^{1}$} \\
\hline & $\%$ & SE & OR & \multicolumn{2}{|c|}{$95 \% \mathrm{CI}$} & $\%$ & SE & OR & \multicolumn{2}{|c|}{$95 \% \mathrm{CI}$} \\
\hline Non-Hispanic White, US-born & 16.16 & 0.02 & 1.00 & \multicolumn{2}{|c|}{ Reference } & 11.67 & 0.01 & 1.00 & \multicolumn{2}{|c|}{ Reference } \\
\hline Non-Hispanic White, immigrant & 13.75 & 0.08 & 0.68 & 0.67 & 0.69 & 15.02 & 0.07 & 1.78 & 1.75 & 1.81 \\
\hline Mexican, US-born & 12.40 & 0.07 & 0.92 & 0.91 & 0.94 & 28.53 & 0.09 & 1.61 & 1.59 & 1.62 \\
\hline Mexican, immigrant & 8.07 & 0.06 & 0.44 & 0.43 & 0.45 & 57.26 & 0.10 & 4.51 & 4.47 & 4.56 \\
\hline Puerto Rican, US-born & 17.56 & 0.15 & 1.07 & 1.04 & 1.09 & 19.25 & 0.14 & 0.92 & 0.90 & 0.94 \\
\hline Puerto Rican, immigrant & 15.18 & 1.09 & 0.61 & 0.51 & 0.73 & 28.87 & 1.29 & 1.82 & 1.57 & 2.10 \\
\hline Cuban, US-born & 9.19 & 0.28 & 0.99 & 0.92 & 1.06 & 20.24 & 0.37 & 1.27 & 1.20 & 1.34 \\
\hline Cuban, immigrant & 18.08 & 0.25 & 0.70 & 0.67 & 0.72 & 29.93 & 0.27 & 3.29 & 3.18 & 3.40 \\
\hline Central/South American, US-born & 6.38 & 0.16 & 0.80 & 0.76 & 0.84 & 27.22 & 0.27 & 1.46 & 1.41 & 1.50 \\
\hline $\begin{array}{l}\text { Central/South American, } \\
\text { immigrant }\end{array}$ & 8.22 & 0.09 & 0.52 & 0.51 & 0.53 & 44.47 & 0.14 & 3.61 & 3.56 & 3.66 \\
\hline Other Hispanics, US-born & 17.69 & 0.21 & 1.19 & 1.16 & 1.23 & 20.25 & 0.20 & 1.28 & 1.24 & 1.32 \\
\hline Other Hispanics, immigrant & 12.10 & 0.38 & 0.64 & 0.59 & 0.68 & 28.60 & 0.48 & 2.38 & 2.25 & 2.53 \\
\hline Non-Hispanic Black, US-born & 18.96 & 0.05 & 1.03 & 1.02 & 1.04 & 22.29 & 0.05 & 1.13 & 1.12 & 1.14 \\
\hline Non-Hispanic Black, immigrant & 8.46 & 0.12 & 0.58 & 0.57 & 0.60 & 26.62 & 0.17 & 1.99 & 1.95 & 2.03 \\
\hline American Indian/AN & 22.03 & 0.19 & 1.32 & 1.29 & 1.35 & 32.73 & 0.21 & 2.20 & 2.15 & 2.25 \\
\hline Asian Indian, US-born & 4.00 & 0.26 & 0.63 & 0.55 & 0.72 & 12.33 & 0.42 & 0.88 & 0.80 & 0.96 \\
\hline Asian Indian, immigrant & 5.77 & 0.11 & 0.59 & 0.57 & 0.62 & 13.61 & 0.15 & 1.83 & 1.77 & 1.88 \\
\hline Chinese, US-born & 5.49 & 0.20 & 0.59 & 0.55 & 0.64 & 9.93 & 0.24 & 0.77 & 0.72 & 0.81 \\
\hline Chinese, immigrant & 7.33 & 0.11 & 0.41 & 0.39 & 0.42 & 16.40 & 0.14 & 1.72 & 1.68 & 1.76 \\
\hline Filipino, US-born & 6.52 & 0.23 & 0.79 & 0.73 & 0.85 & 12.66 & 0.29 & 0.94 & 0.88 & 0.99 \\
\hline Filipino, immigrant & 9.94 & 0.14 & 0.77 & 0.74 & 0.79 & 11.74 & 0.14 & 1.53 & 1.48 & 1.58 \\
\hline Japanese, US-born & 14.77 & 0.33 & 0.87 & 0.83 & 0.92 & 5.24 & 0.18 & 0.68 & 0.62 & 0.74 \\
\hline Japanese, immigrant & 7.51 & 0.30 & 0.41 & 0.38 & 0.44 & 10.02 & 0.30 & 1.25 & 1.15 & 1.35 \\
\hline Korean, US-born & 5.20 & 0.34 & 0.61 & 0.54 & 0.69 & 20.11 & 0.55 & 1.50 & 1.38 & 1.62 \\
\hline Korean, immigrant & 6.80 & 0.16 & 0.41 & 0.39 & 0.44 & 28.06 & 0.27 & 4.06 & 3.94 & 4.20 \\
\hline Vietnamese, US-born & 5.29 & 0.36 & 0.70 & 0.61 & 0.80 & 25.18 & 0.67 & 1.58 & 1.46 & 1.70 \\
\hline Vietnamese, immigrant & 10.25 & 0.19 & 0.57 & 0.54 & 0.59 & 22.34 & 0.24 & 1.76 & 1.70 & 1.82 \\
\hline Cambodian, US-born & 5.43 & 0.76 & 0.69 & 0.51 & 0.92 & 35.22 & 1.56 & 1.44 & 1.24 & 1.67 \\
\hline Cambodian, immigrant & 15.29 & 0.63 & 0.73 & 0.65 & 0.81 & 22.39 & 0.70 & 1.31 & 1.20 & 1.44 \\
\hline Bangladeshi, US-born & 4.74 & 2.17 & 0.53 & 0.20 & 1.38 & 14.77 & 3.91 & 1.10 & 0.65 & 1.87 \\
\hline Bangladeshi, immigrant & 6.74 & 0.62 & 0.53 & 0.43 & 0.66 & 28.14 & 1.08 & 2.37 & 2.10 & 2.67 \\
\hline Pakistani, US-born & 5.04 & 0.79 & 0.64 & 0.43 & 0.95 & 22.23 & 1.62 & 1.59 & 1.30 & 1.94 \\
\hline Pakistani, immigrant & 6.99 & 0.36 & 0.57 & 0.51 & 0.64 & 30.29 & 0.62 & 3.26 & 3.04 & 3.50 \\
\hline Hmong, US-born & 4.28 & 0.65 & 0.59 & 0.43 & 0.82 & 26.57 & 1.44 & 1.15 & 0.99 & 1.34 \\
\hline Hmong, immigrant & 14.37 & 0.83 & 0.74 & 0.64 & 0.85 & 18.40 & 0.88 & 0.81 & 0.71 & 0.92 \\
\hline Laotian, US-born & 4.97 & 0.90 & 0.71 & 0.51 & 1.00 & 33.07 & 1.80 & 1.53 & 1.29 & 1.82 \\
\hline Laotian, immigrant & 12.33 & 0.66 & 0.69 & 0.61 & 0.78 & 17.67 & 0.72 & 1.05 & 0.94 & 1.17 \\
\hline Thai, US-born & 3.45 & 0.85 & 0.56 & 0.35 & 0.90 & 22.97 & 1.84 & 1.53 & 1.22 & 1.94 \\
\hline Thai, immigrant & 6.77 & 0.46 & 0.43 & 0.37 & 0.50 & 24.13 & 0.72 & 2.48 & 2.26 & 2.73 \\
\hline Other Asians, US-born & 5.98 & 0.38 & 0.68 & 0.59 & 0.77 & 13.49 & 0.51 & 0.83 & 0.75 & 0.92 \\
\hline Other Asians, immigrant & 7.04 & 0.24 & 0.53 & 0.49 & 0.57 & 21.04 & 0.36 & 1.73 & 1.64 & 1.82 \\
\hline Native Hawaiian & 15.17 & 0.65 & 1.06 & 0.96 & 1.17 & 13.90 & 0.59 & 0.88 & 0.79 & 0.98 \\
\hline Samoan & 13.49 & 0.91 & 1.04 & 0.88 & 1.23 & 21.38 & 1.05 & 0.98 & 0.86 & 1.13 \\
\hline Guamanian & 11.02 & 0.92 & 0.99 & 0.82 & 1.19 & 19.23 & 1.04 & 1.08 & 0.92 & 1.27 \\
\hline Other Pacific Islanders, US-born & 8.44 & 1.27 & 0.79 & 0.57 & 1.08 & 24.61 & 1.87 & 1.22 & 0.98 & 1.51 \\
\hline
\end{tabular}


TABLE 5: Continued.

\begin{tabular}{|c|c|c|c|c|c|c|c|c|c|c|}
\hline \multirow{4}{*}{$\begin{array}{l}\text { Ethnic immigrant group } \\
\text { Other Pacific Islanders, immigrant }\end{array}$} & \multicolumn{5}{|c|}{ Disability } & \multicolumn{5}{|c|}{ No health insurance } \\
\hline & \multicolumn{2}{|c|}{ Prevalence } & \multicolumn{3}{|c|}{ Adjusted odds ratio ${ }^{1}$} & \multicolumn{2}{|c|}{ Prevalence } & \multicolumn{3}{|c|}{ Adjusted odds ratio ${ }^{1}$} \\
\hline & \multirow{2}{*}{$\begin{array}{c}\% \\
12.61\end{array}$} & \multirow{2}{*}{$\frac{\mathrm{SE}}{1.29}$} & \multirow{2}{*}{$\frac{\mathrm{OR}}{0.64}$} & \multicolumn{2}{|c|}{$95 \% \mathrm{CI}$} & \multirow{2}{*}{$\frac{\%}{25.01}$} & \multirow{2}{*}{$\frac{\mathrm{SE}}{1.62}$} & \multirow{2}{*}{$\frac{\text { OR }}{1.70}$} & \multicolumn{2}{|c|}{$95 \% \mathrm{CI}$} \\
\hline & & & & 0.49 & 0.83 & & & & 1.38 & 2.10 \\
\hline All other groups, US-born & 18.89 & 0.14 & 1.72 & 1.69 & 1.76 & 20.32 & 0.13 & 1.20 & 1.17 & 1.22 \\
\hline All other groups, immigrant & 9.93 & 0.25 & 0.72 & 0.68 & 0.77 & 24.59 & 0.34 & 2.11 & 2.02 & 2.21 \\
\hline
\end{tabular}

OR: odds ratio; SE: standard error; CI: confidence interval.

${ }^{1}$ Adjusted by logistic regression model for age, gender, marital status, education, poverty, and employment status.

TABLE 6: Prevalence and adjusted odds of lack of health insurance among people with disabilities according to race/ethnicity: The 2008-2010 American Community Survey $(N=1,233,595)$.

\begin{tabular}{|c|c|c|c|c|c|c|c|c|c|c|}
\hline \multirow{3}{*}{ Race/ethnicity } & \multicolumn{5}{|c|}{ All ages } & \multicolumn{5}{|c|}{ Age $\geq 18$ years } \\
\hline & \multicolumn{2}{|c|}{ Prevalence } & \multicolumn{3}{|c|}{ Adjusted odds ratio ${ }^{1}$} & \multicolumn{2}{|c|}{ Prevalence } & \multicolumn{3}{|c|}{ Adjusted odds ratio $^{2}$} \\
\hline & $\%$ & SE & OR & \multicolumn{2}{|c|}{$95 \% \mathrm{CI}$} & $\%$ & SE & OR & \multicolumn{2}{|c|}{$95 \% \mathrm{CI}$} \\
\hline Non-Hispanic White & 8.22 & 0.03 & 1.00 & \multicolumn{2}{|c|}{ Reference } & 8.45 & 0.03 & 1.00 & \multicolumn{2}{|c|}{ Reference } \\
\hline Mexican & 22.69 & 0.16 & 1.70 & 1.66 & 1.75 & 24.69 & 0.17 & 1.64 & 1.60 & 1.69 \\
\hline Puerto Rican & 10.14 & 0.24 & 0.78 & 0.73 & 0.82 & 11.32 & 0.27 & 0.76 & 0.72 & 0.81 \\
\hline Cuban & 12.61 & 0.39 & 1.16 & 1.06 & 1.27 & 12.90 & 0.41 & 1.17 & 1.06 & 1.28 \\
\hline Central/South American & 21.98 & 0.34 & 1.42 & 1.35 & 1.50 & 23.62 & 0.37 & 1.33 & 1.26 & 1.40 \\
\hline Other Hispanics & 12.75 & 0.33 & 1.11 & 1.04 & 1.20 & 13.80 & 0.36 & 1.11 & 1.03 & 1.19 \\
\hline Non-Hispanic Black & 13.11 & 0.08 & 1.07 & 1.05 & 1.09 & 14.01 & 0.09 & 1.05 & 1.03 & 1.07 \\
\hline American Indian/AN & 20.01 & 0.34 & 1.84 & 1.75 & 1.93 & 20.84 & 0.36 & 1.84 & 1.75 & 1.94 \\
\hline Asian Indian & 13.14 & 0.57 & 1.23 & 1.09 & 1.38 & 13.84 & 0.61 & 1.39 & 1.24 & 1.57 \\
\hline Chinese & 7.56 & 0.32 & 0.78 & 0.69 & 0.87 & 7.83 & 0.34 & 0.83 & 0.74 & 0.94 \\
\hline Filipino & 7.81 & 0.34 & 0.80 & 0.72 & 0.89 & 7.95 & 0.35 & 0.86 & 0.77 & 0.97 \\
\hline Japanese & 2.28 & 0.26 & 0.47 & 0.35 & 0.63 & 2.26 & 0.26 & 0.50 & 0.37 & 0.66 \\
\hline Korean & 13.31 & 0.67 & 1.12 & 0.96 & 1.29 & 13.73 & 0.70 & 1.23 & 1.06 & 1.42 \\
\hline Vietnamese & 10.47 & 0.51 & 0.63 & 0.56 & 0.72 & 10.59 & 0.53 & 0.65 & 0.57 & 0.73 \\
\hline Cambodian & 13.97 & 1.33 & 0.63 & 0.49 & 0.82 & 14.64 & 1.44 & 0.63 & 0.49 & 0.82 \\
\hline Bangladeshi & 20.09 & 3.37 & 0.98 & 0.60 & 1.60 & 20.92 & 3.68 & 1.05 & 0.63 & 1.76 \\
\hline Pakistani & 25.13 & 1.98 & 1.60 & 1.23 & 2.08 & 27.30 & 2.17 & 1.98 & 1.51 & 2.59 \\
\hline Hmong & 10.43 & 1.59 & 0.35 & 0.24 & 0.51 & 10.06 & 1.68 & 0.36 & 0.25 & 0.53 \\
\hline Laotian & 13.45 & 1.60 & 0.65 & 0.48 & 0.88 & 14.41 & 1.71 & 0.65 & 0.48 & 0.89 \\
\hline Thai & 8.95 & 1.62 & 0.46 & 0.28 & 0.74 & 9.47 & 1.72 & 0.47 & 0.29 & 0.77 \\
\hline Other Asians & 12.45 & 0.92 & 0.80 & 0.66 & 0.96 & 13.40 & 1.01 & 0.80 & 0.66 & 0.96 \\
\hline Native Hawaiian & 9.96 & 1.13 & 0.91 & 0.67 & 1.24 & 10.55 & 1.20 & 0.86 & 0.62 & 1.17 \\
\hline Samoan & 14.36 & 2.48 & 1.12 & 0.76 & 1.64 & 14.79 & 2.61 & 1.08 & 0.72 & 1.63 \\
\hline Guamanian & 8.82 & 2.37 & 1.00 & 0.58 & 1.73 & 8.99 & 2.46 & 0.84 & 0.47 & 1.48 \\
\hline Other Pacific Islanders & 25.86 & 3.50 & 1.47 & 0.92 & 2.35 & 25.94 & 3.64 & 1.33 & 0.82 & 2.16 \\
\hline All other groups & 14.29 & 0.22 & 1.19 & 1.14 & 1.24 & 16.61 & 0.26 & 1.20 & 1.15 & 1.25 \\
\hline
\end{tabular}

${ }^{1}$ Adjusted by logistic regression model for age, gender, immigrant status, and poverty level.

${ }^{2}$ Adjusted by logistic regression model for age, gender, immigrant status, marital status, education, poverty, and employment status.

groups such as Asian Indians, Chinese, Koreans, and Japanese who have immigrated to the US primarily under skill criteria, many of the Southeast Asian immigrants, substantially less educated and much poorer than other Asian Americans, arrived in the USA under the broad refugee and resettlement act of $1980[4,5]$. As shown in Table 1 , they continue to remain greatly disadvantaged socioeconomically, with Hmong having the highest child and adult poverty rates of all groups in the USA. Native Hawaiians, Samoans, and Guamanians, who are also socioeconomically disadvantaged, have higher adult disability rates than many Asian groups, although they do not differ significantly from Whites or Blacks in their disability risks. All Hispanic subgroups have higher child and adult disability rates than most Asian groups, with Puerto Ricans, the most disadvantaged Hispanic group, showing the highest child disability rate in the US. American Indians/Alaska Natives and Blacks show higher rates of child and adult disability rates than Whites and Asian groups. American 


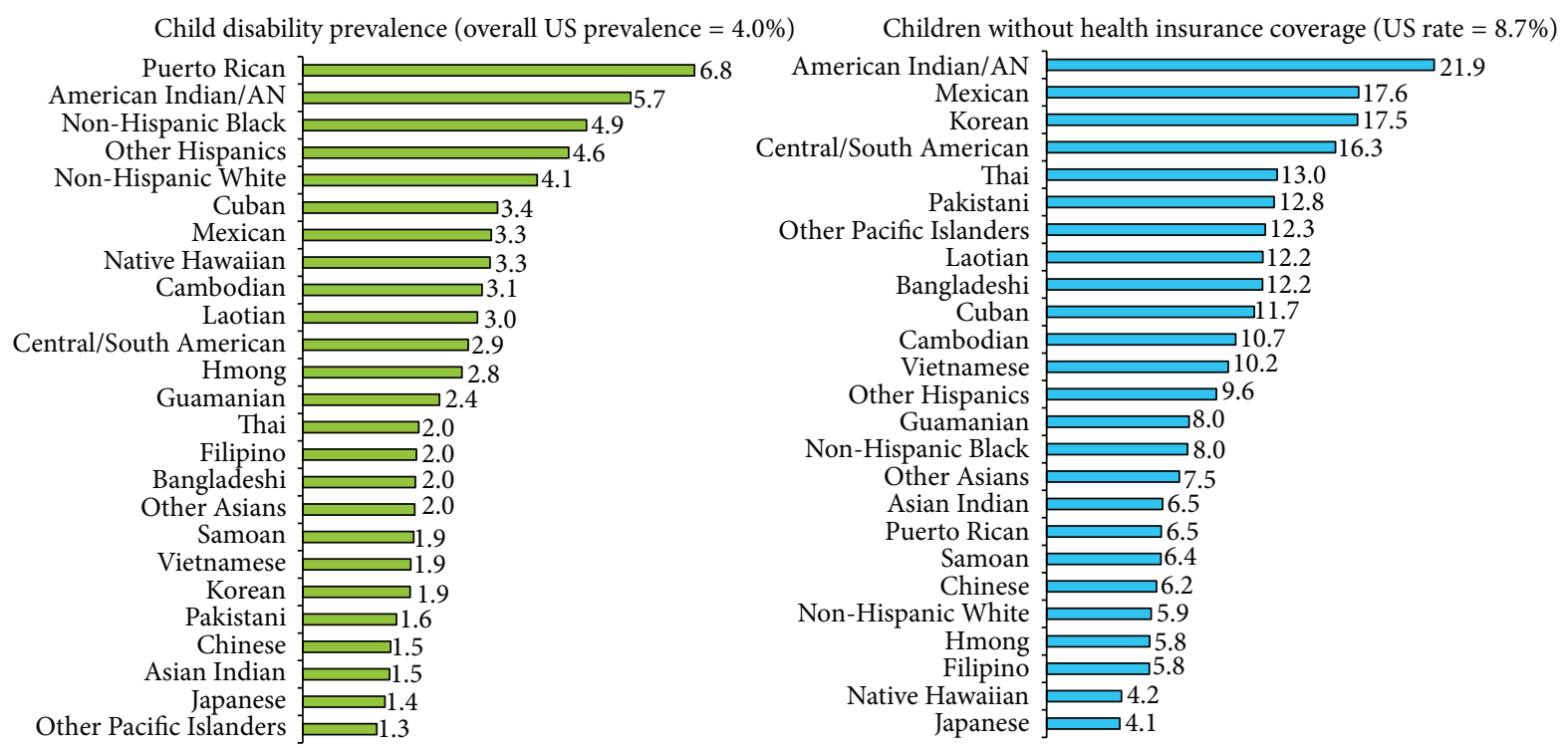

Adult disability prevalence (overall US prevalence $=15.2 \%$ )

Adults without health insurance coverage (US rate $=17.4 \%$ )
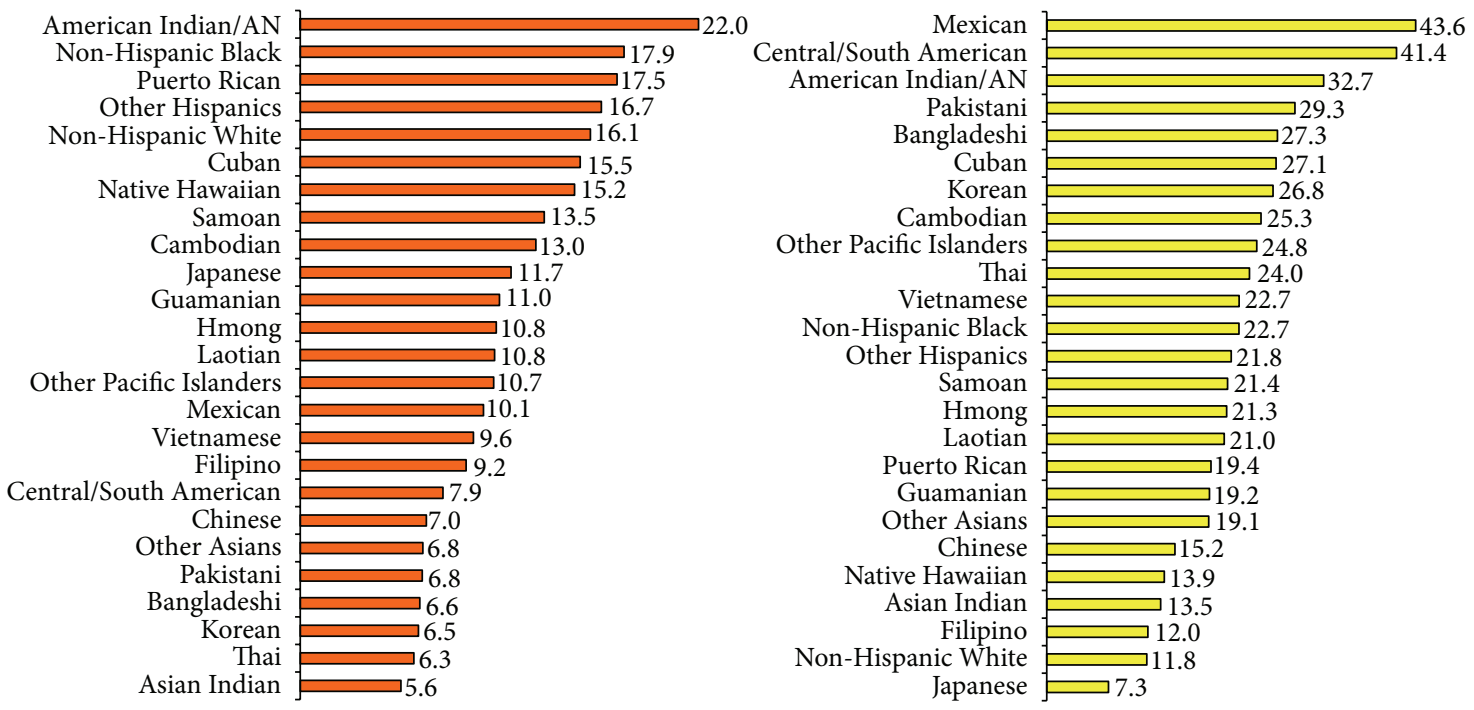

FIGURE 1: Racial/ethnic disparities in the prevalence (\%) of disability and lack of health insurance coverage among US children aged $<18$ and adults aged 18+ years, 2008-2010.

Indians/Alaska Natives and Blacks have long experienced a disadvantaged position in the American society, as they have lagged behind Whites in their socioeconomic attainment, employment, health status, and access to and use of healthcare services $[1,22,35]$. They are more likely to report higher rates of several health-risk behaviors such as smoking, heavy alcohol consumption, substance use, and co-morbid conditions such as obesity, hypertension, diabetes, and cardiovascular diseases, which may contribute to their higher rates of disability [22,31,32,35].

Social inequalities in health insurance coverage were marked, with most of the ethnic inequality attributable to nativity and SES differences. Yet children and adults in several minority and ethnic immigrant groups remained at considerably higher risk of uninsurance compared to
Whites of equivalent SES background. An uninsurance rate of $>55 \%$ for Mexican immigrant children and adults and a rate approaching or exceeding 30\% for some of the US-born and low-SES groups indicate the magnitude of the uninsurance problem across various demographic groups in the US.

Since cognitive/mental difficulties contribute most to disabilities in children, differences in socioeconomic, familial, and behavioral risk factors are most likely to explain racial/ethnic and nativity disparities in child disability rates $[17,19]$. Since many mental and physical health conditions that cause various disabilities require a doctor's or health-care provider's diagnosis, the substantially lower rates of health insurance, healthcare access, and healthcare utilization or interaction with the healthcare system among immigrant and ethnic minority groups such as Asians, Hispanics, Blacks, 

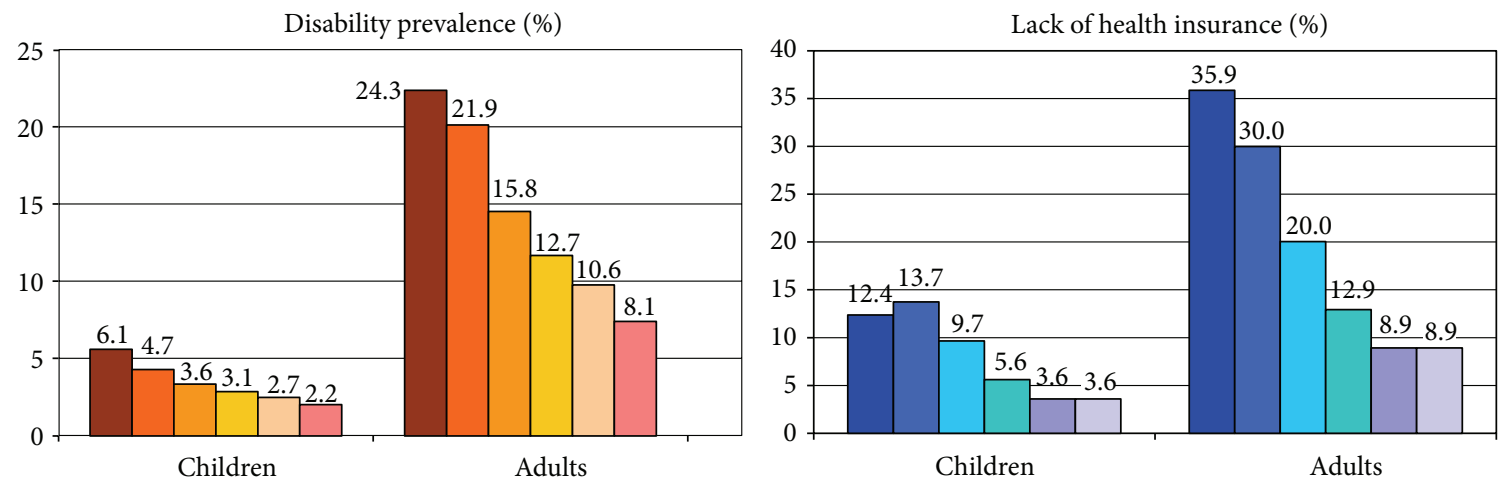
- Poverty level $<100 \%$
$300-399 \%$
ㅁ 100-199\%
ㅁ $400-499 \%$
$200-299 \%$
$\geq 500 \%$
- Poverty level $<100 \%$
ㅁ 300-399\%
- 100-199\%
ㅁ $400-499 \%$
- 200-299\%
ㅁ $\geq 500 \%$

FIGURE 2: Disparities in prevalence (\%) of disability and lack of health insurance coverage among US children aged $<18$ years and adults aged $18+$ years according to poverty level. Notes: Differences in prevalence of disability and health insurance across poverty categories were statistically significant at $P<0.001$. Source: [36].

Child disability prevalence (US prevalence $=4.0 \%$ )

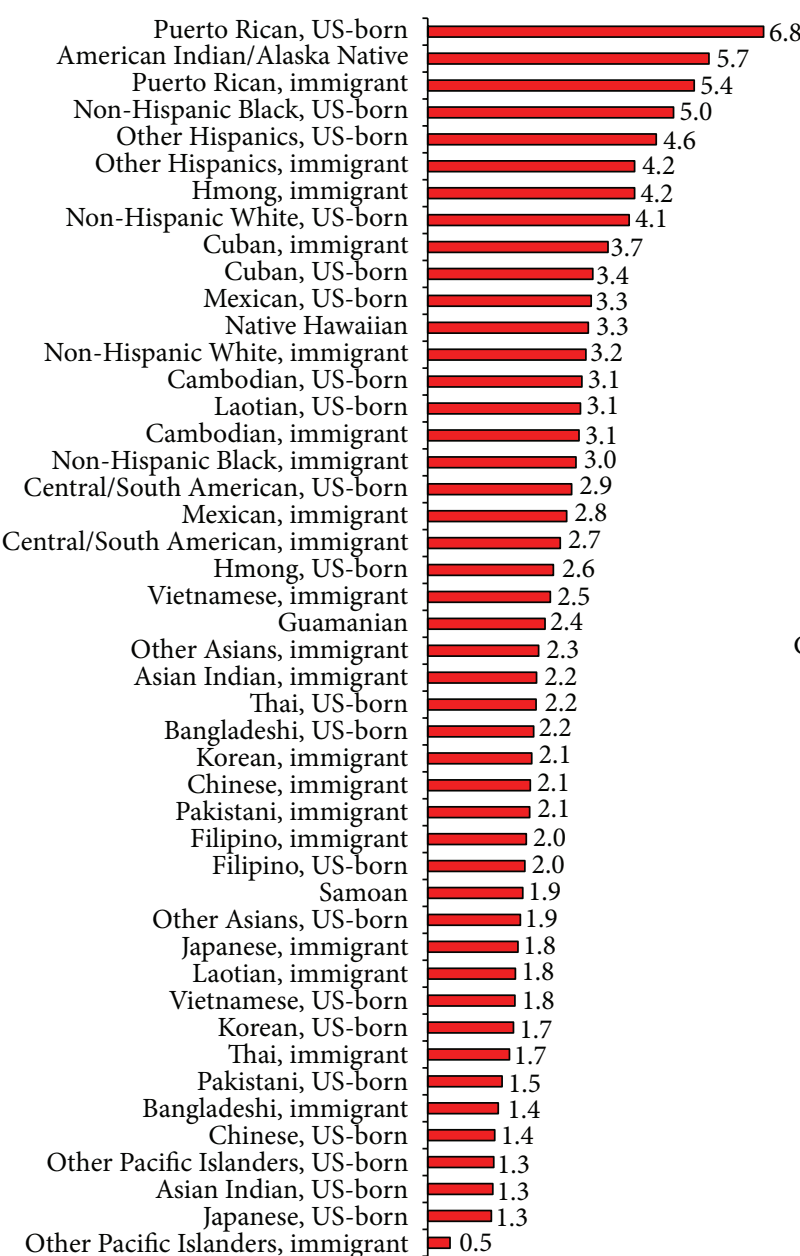

Adult disability prevalence (US prevalence $=15.2 \%$ )

American Indian/Alaska Native Non-Hispanic Black, US-born Cuban, immigrant

Other Hispanics, US-born Puerto Rican, US-born Non-Hispanic White, US-born

Cambodian, immigrant

Puerto Rican, immigrant Native Hawaiian Japanese, US-born Hmong, immigrant Non-Hispanic White, immigrant Samoan

Other Pacific Islanders, immigrant

Mexican, US-born

Laotian, immigrant

Other Hispanics, immigran Guamanian

Vietnamese, immigrant

Filipino, immigrant Cuban, US-born

Non-Hispanic Black, immigran Other Pacific Islanders, US-born Central/South American, immigrant Mexican, immigrant Japanese, immigrant Chinese, immigrant

Other Asians, immigrant

Pakistani, immigran

Korean, immigran Thai, immigrant

Bangladeshi, immigrant Filipino, US-born Central/South American, US-born

Other Asians, US-born

Asian Indian, immigrant

Chinese, US-born

Cambodian, US-born

Vietnamese, US-born

Korean, US-born

Pakistani, US-born

Laotian, US-born

Bangladeshi, US-born

Hmong, US-born

Asian Indian, US-born Thai, US-born

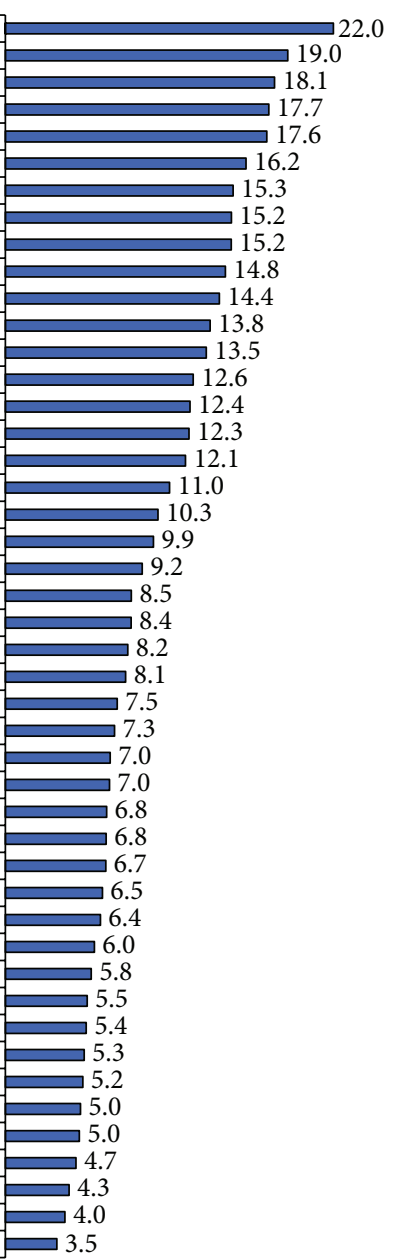

FIGURE 3: Ethnic immigrant disparities in the prevalence (\%) of disability among US children aged $<18$ years and adults aged $18+$ years, 2008-2010. 

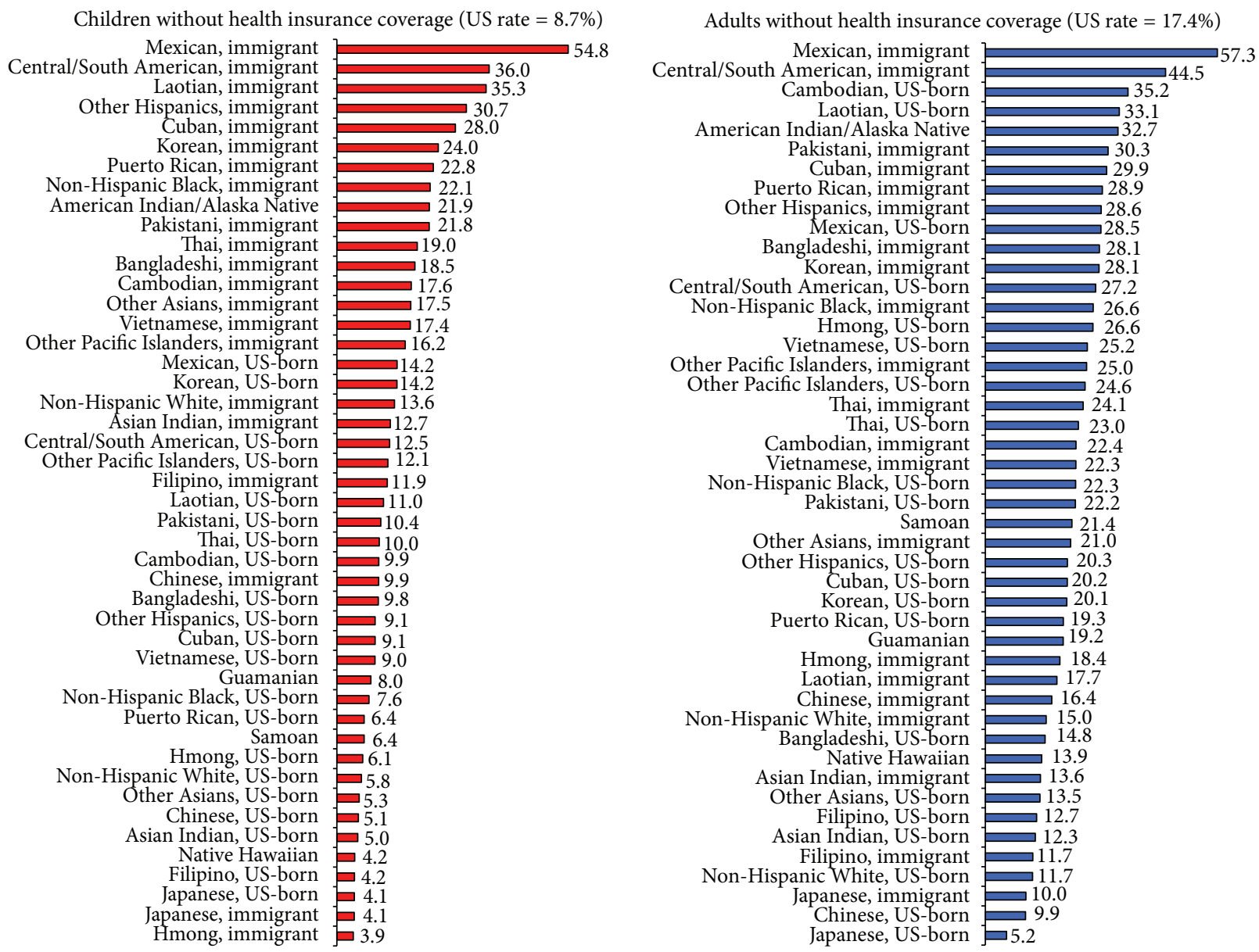

FIGURE 4: Ethnic immigrant variations in lack of health insurance coverage (\%) among US children aged $<18$ years and adults aged $18+$ years, 2008-2010.

and American Indians/Alaska Natives might result in fewer diagnoses of disability-related health conditions among them and could partly account for the racial/ethnic disparities in disability rates reported here. Asian and Hispanic subgroups vary greatly in their cultures, English language proficiencies, and perhaps in their interpretation/understanding of the disability questions in the ACS, all of which can also contribute to the reported ethnic differences in disability rates. Among people with disabilities, American Indians/Alaska Natives, Hispanics, Asians, and several immigrant groups have substantially high rates of uninsurance, which may imply that they are more likely to delay or not receive needed medical care, preventive health services, social, and/or rehabilitative services [22, 23].

Ethnic and nativity patterns in disability are consistent with those observed for a wide range of health outcomes $[11,12,22,29,31,32,42]$. In the USA, the major Asian and Hispanic subgroups, including Chinese, Asian Indians, Filipinos, Japanese, Mexicans, Cubans, and Central/South Americans have higher life expectancy and lower rates of all-cause, chronic disease, and injury mortality and morbidity than Whites, Blacks, and American Indians/and Alaska Natives $[11,12,22,29,31,32]$. Consistent with the patterns in disability, immigrants, overall and in most racial/ethnic groups, do better than the US-born in various child and adult health outcomes [11, 12, 29, 42]. Healthy immigrant effect or positive immigrant selectivity (i.e., people immigrating to the USA may be healthier than those who remain in their countries of origin) has been offered as an explanation of better health and lower disability and mortality rates of immigrants [11-13, 29, 42]. Acculturation, often measured by duration of residence since the time of immigration, is also shown to play a part in modifying the risks of disability and health insurance among immigrants [11-13]. However, lower disability rates among individuals in many of the US-born Asian and Hispanic subgroups compared with US-born Whites may offer support for the cultural pluralism hypothesis, which contends that many groups retain significant ethnic and social ties to their cultural heritage across generations in the host country [11, 12, 43].

Our study has limitations. Although the ACS summary database does include the type of disability (sensory, mental, and physical) for both children and adults, the micro-data sample lacks data on both the type and severity of disabilities $[2,18,19,36]$. Social and ethnic patterns might vary according to disability type and may be more pronounced 
in physical than mental health disabilities [13-19]. The ACS also excludes data on institutionalized populations, such as those in prisons, nursing homes, and military who may have different disability and uninsurance rates than the general population [36-38]. Additionally, although the ACS does include a number of immigration-related variables such as citizenship/naturalization status, English language ability, length of US residence, and age at entry into the US, it does lack data on the legal status of immigrants which could greatly influence their access to health insurance [36-38]. Lastly, the ACS is a cross-sectional database, and disability can be both a cause and consequence of social and economic disadvantage [25, 36, 37].

Although the definition of disability varies somewhat across the developed world, the disability rates in the USA are comparable to those in Canada, Australia, and the United Kingdom [21, 25, 44, 45]. However, the social, economic, and labor force experiences of people with disabilities, and disability policies vary greatly among nations. According to a recent Organisation for Economic Cooperation and Development (OECD) study, young Americans aged 18-29 with a disability were $80 \%$ less likely to be employed than their counterparts without a disability [45]. The ACS data show an adult employment rate of $21.8 \%$ for people with disabilities, compared with $64.2 \%$ for people without a disability [2]. Of the 25 OECD countries, the gap in employment by disability status and the poverty rate for households with a disabled child were the highest in the USA [45]. The USA ranks poorly in its social and economic inclusion of people with disabilities and in its disability benefit, compensation, and integration policies compared to most other OECD nations, particularly the Nordic countries [45].

Children with disabilities from immigrant families confront challenges in access to high-quality medical care due to lack of parental awareness in eligibility criteria for safetynet programs and insurance and nativity status of the parent $[46,47]$. Policymakers have made advances in children's health insurance coverage by passing the Children's Health Insurance Program Reauthorization Act (CHIPRA) of 2009, which granted states an option to provide federally funded Medicaid and CHIP coverage for lawfully residing children and pregnant women. Although twenty-three states and the District of Columbia are using this option to offer coverage to legal immigrant children without a five-year waiting period, children residing in states that have not elected to implement eligibility expansion or to simplify enrollment and renewal procedures remain at risk for uninsurance [48]. Inadequate access to quality care for these children warrants further policy solutions to improve their health care utilization, especially in obtaining culturally sensitive care, communitybased support, and advocacy for services [49-51].

For adults, the Affordable Care Act of 2010 will offer options for legal immigrants to purchase affordable coverage through the Health Insurance Marketplace (also known as the Exchange). Initial provision of the Affordable Care Act has provided coverage to millions of young adults by permitting them to stay on their parents' health plan until age 26 and children with pre-existing conditions by requiring insurers to no longer exclude, limit, or deny coverage to children under age 19 solely based on a health problem or disability [52]. Moreover, the Health Insurance Marketplace will be a new pathway to purchase health insurance beginning on October 1, 2013. Families will be able to get financial assistance through the Health Insurance Marketplace. There will be new, expanded programs available, and more people than ever before will qualify for free or low-cost health insurance programs. Concurrently, the federally funded Marketplace Navigators program will provide culturally and linguistically appropriate consumer information and assistance regarding public and private insurance coverage to diverse communities and people with disabilities [53]. This program will provide critical family support in navigation through the Health Insurance Marketplace.

The findings presented here demonstrate considerable heterogeneity in disability and insurance status among racial/ethnic, immigrant, and socioeconomic groups. While the provisions of the Affordable Care Act hold promise for expanding coverage to those currently uninsured, targeted and culturally competent outreach and enrollment programs for the Health Insurance Marketplace will be critical in raising public awareness as racial/ethnic and immigrant groups may have different levels of awareness and/or understanding about benefits and eligibility criteria of health insurance plans and safety net programs. The successful outreach of the Marketplace Navigators and other in-person assistance programs through initiating new or enhancing existing partnerships with ethnic immigrant community-based organizations will greatly benefit ethnic immigrant groups, especially individuals and children with disabilities.

\section{Disclosure}

No IRB approval was required for this study, which is based on the secondary analysis of a public-use federal database.

\section{Disclaimer}

The views expressed are the authors' and not necessarily those of the Health Resources and Services Administration or the US Department of Health and Human Services.

\section{Conflict of Interests}

The authors declare that there is no conflict of interests.

\section{References}

[1] US Census Bureau, Statistical Abstract of the United States, Government Printing Office, Washington, DC, USA, 131th edition, 2012.

[2] US Census Bureau, American Community Survey, Government Printing Office, Washington, DC, USA, 131th edition, 2012.

[3] J. D. Pinal and S. J. Lapham, We, the American Hispanics, US Census Bureau, Washington, DC, USA, 1993.

[4] D. L. Johnson, M. J. Levin, and E. L. Paisano, We, the Asian and Pacific Islander Americans, US Census Bureau, Washington, DC, USA, 1988. 
[5] Pew Research Center, The Rise of Asian Americans, Pew Social \& Demographic Trends, Washington, DC, USA, 2012.

[6] L. P. Walters and E. N. Trevelyan, The Newly Arrived ForeignBorn Population of the United States: 2010, American Community Survey Briefs, US Census Bureau, Washington, DC, 2011.

[7] E. M. Grieco and E. N. Trevelyan, Place of Birth of the ForeignBorn Population: 2009, American Community Survey Briefs, US Census Bureau, Washington, DC, USA, 2010.

[8] E. M. Grieco, "Race and hispanic origin of the foreign-born population in the United States: 2007," American Community Survey Reports, US Census Bureau, Washington, DC, USA, 2010.

[9] L. J. Larsen, "The Foreign-Born Population in the United States: March 2003," Current Population Reports, US Census Bureau, Washington, DC, USA, 2004.

[10] Federal Interagency Forum on Child and Family Statistics, America'S Children: Key National Indicators of Well-Being, US Government Printing Office, Washington, DC, USA, 2012.

[11] G. K. Singh and B. A. Miller, "Health, life expectancy, and mortality patterns among immigrant populations in the United States," Canadian Journal of Public Health, vol. 95, no. 3, pp. I14I21, 2004.

[12] G. K. Singh and R. A. Hiatt, "Trends and disparities in socioeconomic and behavioural characteristics, life expectancy, and cause-specific mortality of native-born and foreign-born populations in the United States, 1979-2003," International Journal of Epidemiology, vol. 35, no. 4, pp. 903-919, 2006.

[13] Y. Cho and R. A. Hummer, "Disability status differentials across fifteen asian and pacific islander groups and the effect of nativity and duration of residence in the U.S," Social Biology, vol. 48, no. 3-4, pp. 171-195, 2001.

[14] C. Huang, N. K. Mehta, I. T. Elo et al., "Region of birth and disability among recent U.S. immigrants: evidence from the 2000 Census," Population Research and Policy Review, vol. 30, no. 3, pp. 399-418, 2011.

[15] J. E. Mutchler, A. Prakash, and J. A. Burr, "The demography of disability and the effects of immigrant history: older Asians in the United States," Demography, vol. 44, no. 2, pp. 251-263, 2007.

[16] E. Fuller-Thomson, S. Brennenstuhl, and M. Hurd, "Comparison of disability rates among older adults in aggregated and separate Asian American/Pacific Islander subpopulations," American Journal of Public Health, vol. 101, no. 1, pp. 94-100, 2011.

[17] J. Waldrop and S. M. Stern, Disability Status: 2000. Census 2000 Brief, US Census Bureau, Washington, DC, USA, 2003.

[18] M. W. Brault, Disability Among the Working Age Population: 2008 and 2009, American Community Survey Briefs, US Census Bureau, Washington, DC, USA.

[19] M. W. Brault, School-Aged Children With Disabilities in U.S. Metropolitan Statistical Areas: 2010, American Community Survey Briefs, US Census Bureau, Washington, DC, USA, 2011.

[20] US Census Bureau, "2000 Census of Population and Housing, Summary File 3," Technical Documentation, US Department of Commerce, Washington, DC, USA, 2003.

[21] World Health Organization and The World Bank, World Report on Disability, WHO, Geneva, Switzerland, 2011.

[22] National Center for Health Statistics, Health, United States, 2011 with Special Feature on Socioeconomic Status and Health, US Department of Health and Human Services, Hyattsville, Md, USA, 2012.
[23] B. Altman and A. Bernstein, Disability and Health in the United States, 2001-2005, National Center for Health Statistics, Hyattsville, Md, USA, 2011.

[24] B. W. Ward and J. S. Schiller, "Prevalence of complex activity limitations among racial/ethnic groups and Hispanic subgroups of adults: United States, 2003-2009," NCHS Data Brief, vol. 73, pp. 1-8, 2011.

[25] VicHealth, Disability and Health Inequalities in Australia, Victoria Health Promotion Foundation, Victoria, Australia, 2012, http://www.vichealth.vic.gov.au/publications.

[26] A. P. Wilper, S. Woolhandler, K. E. Lasser, D. McCormick, D. H. Bor, and D. U. Himmelstein, "Health insurance and mortality in US adults," American Journal of Public Health, vol. 99, no. 12, pp. 2289-2295, 2009.

[27] J. M. McWilliams, A. M. Zaslavsky, E. Meara, and J. Z. Ayanian, "Health insurance coverage and mortality among the nearelderly," Health Affairs, vol. 23, no. 4, pp. 223-233, 2004.

[28] F. Abdullah, Y. Zhang, T. Lardaro et al., "Analysis of 23 million US hospitalizations: uninsured children have higher all-cause in-hospital mortality," Journal of Public Health, vol. 32, no. 2, pp. 236-244, 2010.

[29] G. K. Singh and M. Siahpush, "All-cause and cause-specific mortality of immigrants and native born in the United States," American Journal of Public Health, vol. 91, no. 3, pp. 392-399, 2001.

[30] G. K. Singh and M. Siahpush, "Widening socioeconomic inequalities in US life expectancy, 1980-2000," International Journal of Epidemiology, vol. 35, no. 4, pp. 969-979, 2006.

[31] J. S. Schiller, J. W. Lucas, B. W. Ward, and J. A. Peregoy, "Summary health statistics for U.S. adults: National Health Interview Survey, 2010," Vital and Health Statistics, vol. 10, no. 252, pp. 1-207, 2012.

[32] P. F. Adams, M. E. Martinez, J. L. Vickerie, and W. K. Krizinger, "Summary health statistics for the U.S. population: National Health Interview Survey, 2010," Vital and Health Statistics, vol. 10, no. 251, pp. 1-117, 2011.

[33] S. E. Ramsay, P. H. Whincup, R. W. Morris, L. T. Lennon, and S. G. Wannamethee, "Extent of social inequalities in disability in the elderly: results from a population-based study of British men," Annals of Epidemiology, vol. 18, no. 12, pp. 896-903, 2008.

[34] L. M. Verbrugge and A. M. Jette, "The disablement process," Social Science and Medicine, vol. 38, no. 1, pp. 1-14, 1994.

[35] US Department of Health and Human Services, "Healthy people 2020," 2013, http://www.healthypeople.gov/2020/default .aspx.

[36] US Census Bureau, The American Community Survey 2008-2010 ACS 3-Year PUMS Files and Data Documentation, US Census Bureau, Washington, DC, 2011, http://www.census.gov/acs/ www/.

[37] USCensus Bureau, A Compass Ffor Understanding and Using American Community Survey Data: What Researchers Need to Know, US Census Bureau, Washington, DC, USA, 2009.

[38] US Census Bureau, The American Community Survey Design and Methodology Report, US Census Bureau, Washington, DC, USA, 2013, http://www.census.gov/acs/www/methodology/ methodology_main/.

[39] R. Wilkinson and M. Marmot, Eds., Social Determinants of Health: the Solid Facts, World Health Organization, Regional Office for Europe, Copenhagen, Denmark, 2nd edition, 2003.

[40] SAS Institute, SAS/STAT USer'S Guide, VerSion 9.1: the LOGISTIC Procedure, SAS Institute Inc, Cary, NC, USA, 2004. 
[41] SUDAAN, Software for the Statistical Analysis of Correlated Data, Release 10. 0. 1., Research Triangle Institute, Research Triangle Park, NC, USA, 2009.

[42] G. K. Singh and S. M. Yu, "The impact of ethnic-immigrant status and obesity-related risk factors on behavioral problems among US children and adolescents," Scientifica, vol. 2012, Article ID 648152, 14 pages, 2012.

[43] H. J. Gans, "Toward a reconciliation of "assimilation" and "pluralism": the interplay of acculturation and ethnic retention," International Migration Review, vol. 31, no. 4, pp. 875-892, 1997.

[44] Human Resources and Skills Development Canada, Disability in Canada: A, 2006 Profile, Human Resources and Skills Development Canada, Quebec, Canada, 2011, http://www12.hrsdc.gc .ca.

[45] Organisation for Economic Co-operation and Development, Sickness, Disability and Work: Breaking the Barriers. A Synthesis of Findings Across OECD Countries, OECD, Paris, France, 2010.

[46] J. E. DeVoe, C. J. Tillotson, and L. S. Wallace, "Childrens receipt of health care services and family health insurance patterns," Annals of Familyy Medicine, vol. 7, no. 5, pp. 406-412, 2009.

[47] H. Yoshikawa and A. Kalil, "The effects of parental undocumented status on the developmental contexts of young children in immigrant families," Child Development Perspectives, vol. 5, no. 4, pp. 291-297, 2011.

[48] Connecting Kids to Coverage: Steady Growth and New Innovation, CHIPRA Annual Report, 2011, http://insurekidsnow.gov/.

[49] S. Lindsay, G. King, A. F. Klassen, V. Esses, and M. Stachel, "Stachel M. Working with immigrant families raising a child with a disability: challenges and recommendations for healthcare and community service providers," Disability and Rehabilitation, vol. 34, no. 23, pp. 2007-2017, 2012.

[50] S. C. Lin, S. M. Yu, and R. L. Harwood, "Autism spectrum disorders and developmental disabilities in children from immigrant families in the United States," Pediatrics, vol. 130, supplement 2, pp. S191-S197, 2012.

[51] K. Pitkin Derose, B. W. Bahney, N. Lurie, and J. J. Escarce, "Immigrants and health care access, quality, and cost," Medical Care Research and Review, vol. 66, no. 4, pp. 355-408, 2009.

[52] Affordable Care Act: the New Health Care Law at Two Years, http://www.whitehouse.gov/sites/default/files/uploads/ careact.pdf.

[53] New Funding Opportunity Announcement for Navigators in Federally-facilitated and State Partnership Marketplaces, http://cciio.cms.gov/resources/factsheets/navigator-foa.html. 


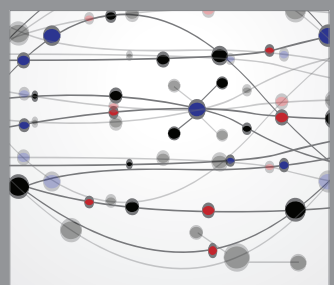

The Scientific World Journal
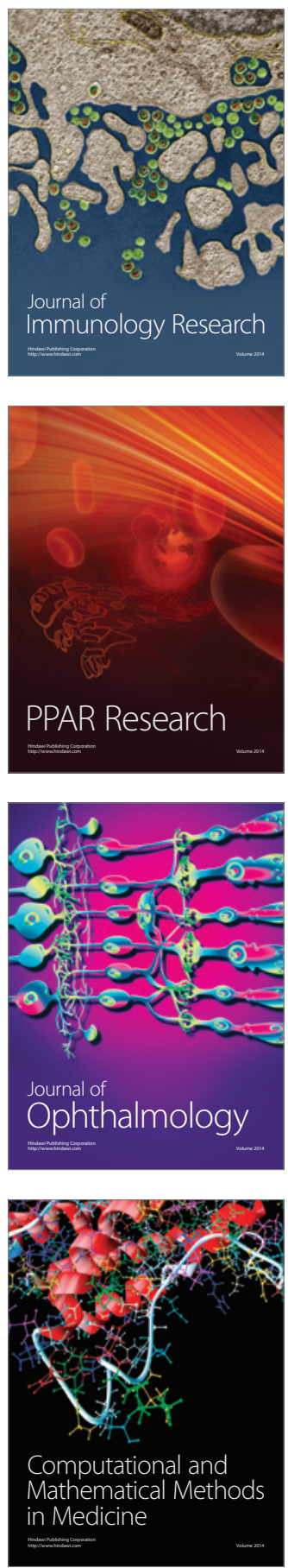

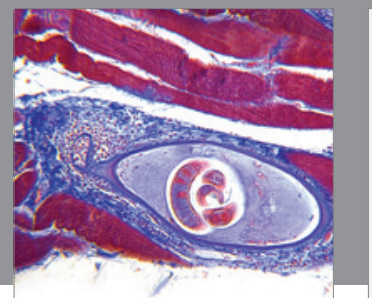

Gastroenterology

Research and Practice
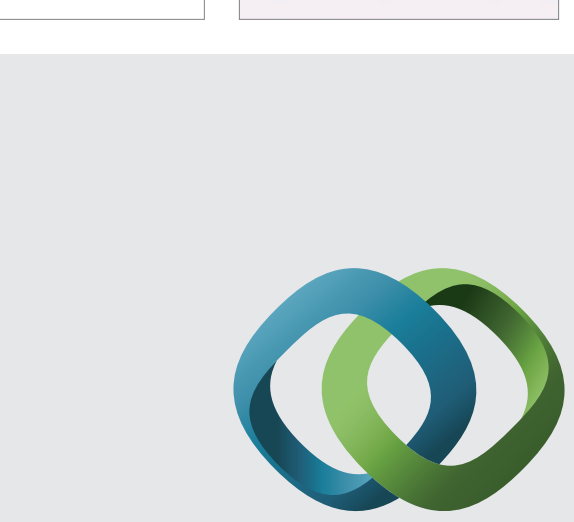

\section{Hindawi}

Submit your manuscripts at

http://www.hindawi.com
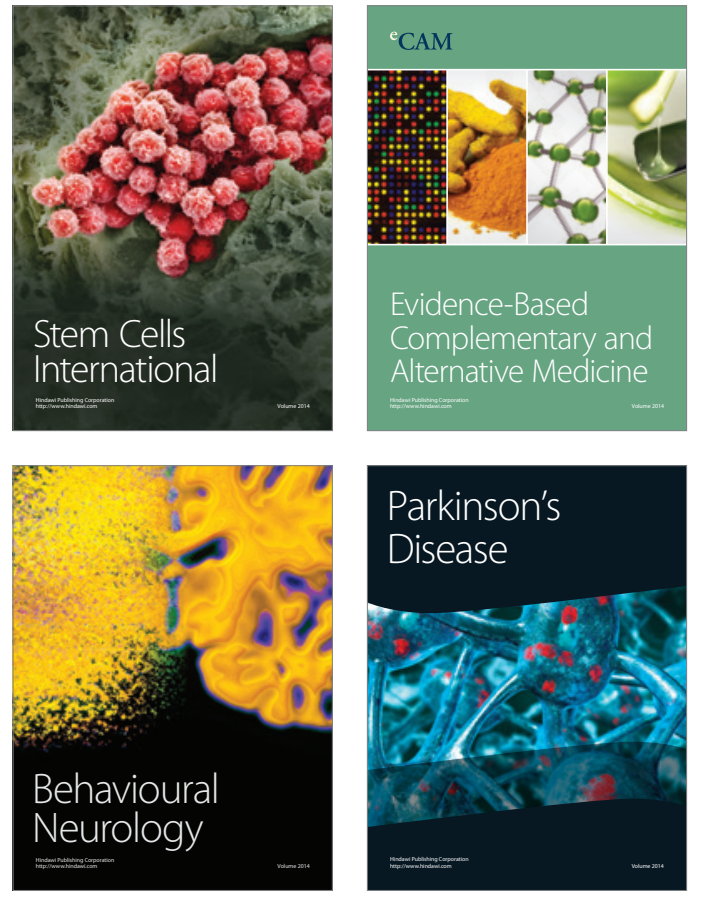
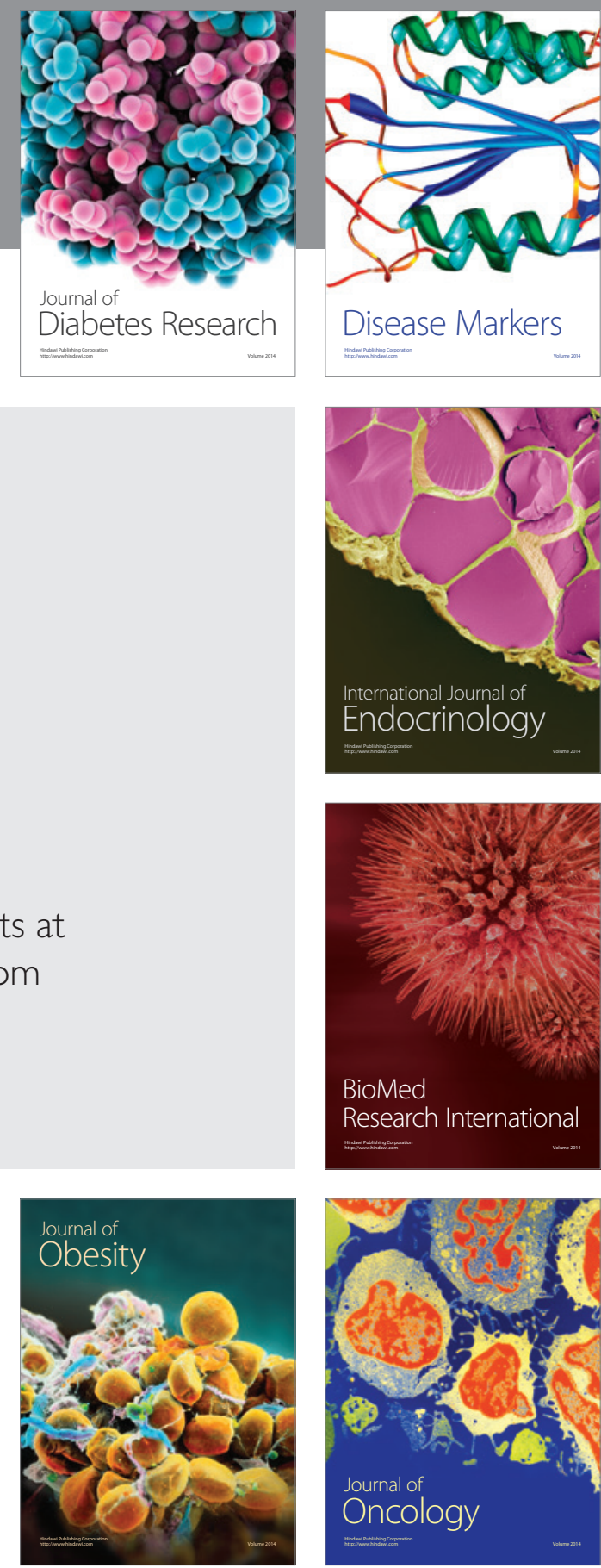

Disease Markers
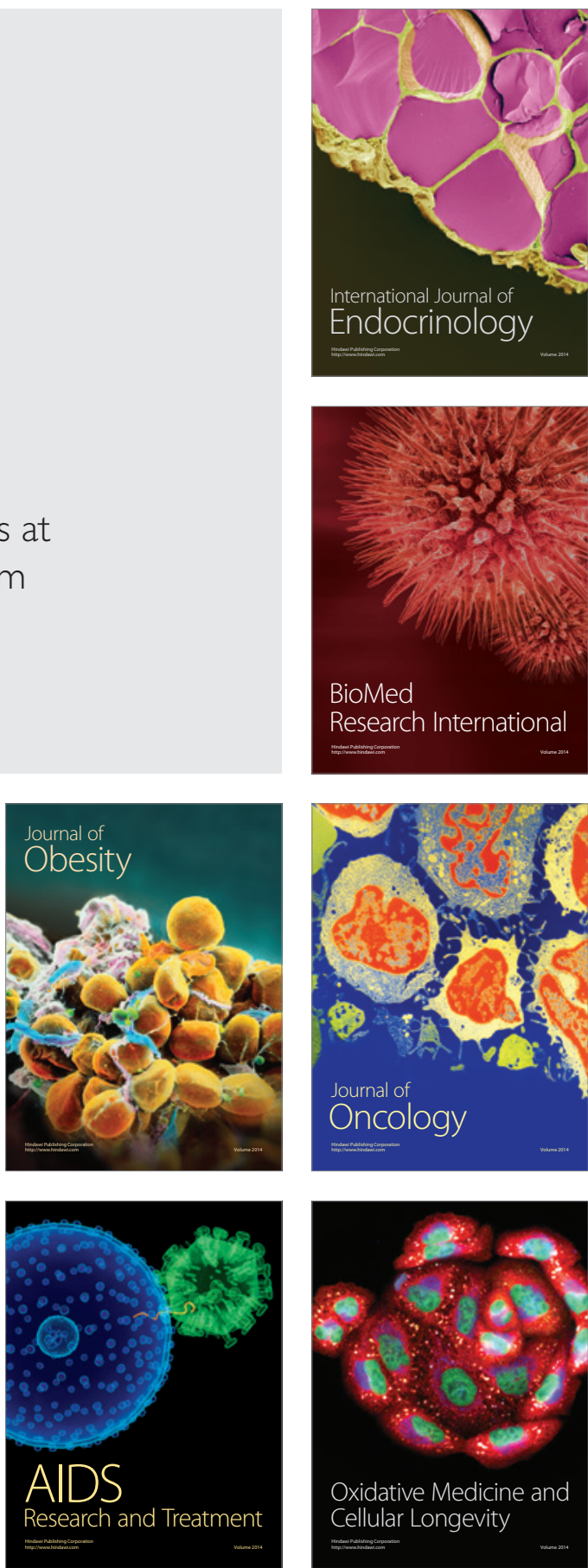